\title{
Received-Signal-Strength Threshold Optimization Using Gaussian Processes
}

\author{
Feng Yin, Yuxin Zhao, Fredrik Gunnarsson and Fredrik Gustafsson \\ Journal Article
}

\section{Tweet}

N.B.: When citing this work, cite the original article.

C2016 IEEE. Personal use of this material is permitted. However, permission to reprint/republish this material for advertising or promotional purposes or for creating new collective works for resale or redistribution to servers or lists, or to reuse any copyrighted component of this work in other works must be obtained from the IEEE.

Feng Yin, Yuxin Zhao, Fredrik Gunnarsson and Fredrik Gustafsson, Received-Signal-Strength Threshold Optimization Using Gaussian Processes, IEEE Transactions on Signal Processing, 2017. 65(8), pp. 2164-2177.

http://dx.doi.org/10.1109/TSP.2017.2655480

Postprint available at: Linköping University Electronic Press

http://urn.kb.se/resolve?urn=urn:nbn:se:liu:diva-135065 


\title{
Received-Signal-Strength Threshold Optimization Using Gaussian Processes
}

\author{
Feng Yin, Member, IEEE, Yuxin Zhao, Student Member, IEEE, Fredrik Gunnarsson, Senior Member, IEEE, \\ Fredrik Gustafsson, Fellow, IEEE
}

\begin{abstract}
There is a big trend nowadays to use event-triggered proximity report for indoor positioning. This paper presents a generic received-signal-strength (RSS) threshold optimization framework for generating informative proximity reports. The proposed framework contains five main building blocks, namely the deployment information, RSS model, positioning metric selection, optimization process and management. Among others, we focus on Gaussian process regression (GPR) based RSS models and positioning metric computation. The optimal RSS threshold is found through minimizing the best achievable localization root-mean-square-error formulated with the aid of fundamental lower bound analysis. Computational complexity is compared for different RSS models and different fundamental lower bounds. The resulting optimal RSS threshold enables enhanced performance of new fashioned low-cost and low-complex proximity report based positioning algorithms. The proposed framework is validated with real measurements collected in an office area where bluetooth-low-energy (BLE) beacons are deployed.
\end{abstract}

Index Terms-Gaussian process, indoor positioning, proximity report, received-signal-strength, threshold optimization.

\section{INTRODUCTION}

\section{A. Background}

Over the past few years, indoor localization and tracking using wireless networks has received considerable attention due to the ever increasing demand on location-awareness in various sectors. So far, most of the efforts have been made to improve the localization accuracy using advanced technologies, for instance statistical sensor fusion [3], dedicated to optimally fuse different types of position-related measurements collected from indoor wireless infrastructures (for instance, cellular, wireless fidelity (Wi-Fi) and bluetooth low-energy (BLE) nodes) and mobile devices. Due to the rapid development of the beaconing techniques, there is a big trend nowadays to use event-triggered proximity information for developing new-fashioned, low-cost (e.g., less communication overhead, smaller database for storage, cheaper deployment and maintenance) indoor positioning systems.

One way of obtaining a proximity report from the network is to compare an instantaneous RSS value with a tuned threshold $P_{t h}$. A proximity report obtained in such way indicates

This work is funded by the European Union FP7 Marie Curie training programme on Tracking in Complex Sensor Systems (TRAX) with grant number 607400. This work is an extension of our conference paper [1] and [2].

F. Yin and Y. Zhao and F. Gunnarsson are with Ericsson Research, Linköping, SE-58330, Sweden. (E-mail: firstname.lastname@ericsson.com).

F. Gustafsson is with the Department of Electrical Engineering, Division of Automatic Control, Linköping University, Linköping, SE-58183, Sweden. (E-mail: fredrik@isy.liu.se). whether or not a user equipment (UE) is in proximity of a reference network node. Essentially, a proximity measurement can be treated as a quantized RSS with merely two quantization levels [3, 4]. Unlike in the conventional paradigm, where the UE sends the measured RSS indication values periodically to the core network, a proximity report is triggered only when the UE's status changes, for instance when the UE is crossing a border to another service region. Such proximity reporting scheme is beneficial in various ways. Among other benefits, the signaling between the UE and the core network can be significantly reduced by sending much less frequently 1-bit proximity values instead of 6-8 bits RSS indication values.

In order to explain the concept of proximity based noncooperative indoor positioning more clearly, we give an illustrative example in Fig. 1, wherein we assume noise free RSS measurements and a simplistic propagation model with which an RSS threshold corresponds to a circular coverage area in open space. As we can see from the figure, the service area is divided into several small regions. An instantaneous RSS measurement being larger than a predefined threshold implies that the UE resides in the corresponding coverage area. For instance, the proximity vector, e.g., $[1,1,0]$, indicates that the $\mathrm{UE}$ is in the coverage area of the first and the second reference nodes, while outside of the coverage area of the third reference node. The UE needs to upload the proximity vector only if there is status change in the proximity report, for instance from $[1,1,0]$ to $[0,1,0]$, when the UE moved from the marked place some distance to the right. However, we note that in practice the RSS measurements are subject to various types of noise and we resort to a statistical framework for RSS thresholding.

\section{B. Related Work and Our Contributions}

In the literature, the proximity based positioning algorithms are often called coarse grained algorithms or range-free algorithms. Since the publication of [4], a plethora of proximity based positioning algorithms have been proposed, including the centroid algorithm [4], the approximate point in triangle (APIT) algorithm [5], the maximum-likelihood estimation based algorithm [6], the ecolocation algorithm [7], and the iterative learning based algorithm [8], to mention a few. The majority of the existing work considered large-scale cooperative sensor network localization subject to communication constraints. To the best of our knowledge, RSS thresholding was first considered for cooperative localization in [9]. Therein, a single RSS threshold is optimized so as to limit the number of the neighboring senors. In our recent work [1], RSS thresholding was considered for non-cooperative, infrastructure-based 
indoor positioning, which can be regarded as a special case of [9]. But the focus of [1] lies in the overall positioning performance in a given service area and thorough treatment on the measurement campaign, RSS modeling, model fitting and parameter calibration, signaling, and performance evaluation using real data measured from a live network. In this work, we extend [1] to multiple RSS thresholds tuning. The performance metric to be optimized is selected to be the overall positioning root-mean-square-error (RMSE) represented in terms of the Cramér-Rao bound or Barankin bound [2]. The former bound is suitable to benchmark estimation performance for mediumand large scale sensor networks, while the latter bound is more suitable to benchmark small scale sensor networks. Moreover, we introduce advanced Gaussian process regression (GPR) based RSS models, perform detailed performance analyses and validate the results with more real data. Lastly, we incorporate the derived fundamental lower bounds and the advanced GPR based RSS models into RSS thresholds optimization. To give a quick overview, the proposed generic framework for selecting a set of reasonable RSS thresholds for proximity report based positioning is given in Figure 2.

\section{Paper Organization and Notations}

The remainder of this paper is organized as follows: Section II lists the prerequisites for performing the RSS threshold optimization, including the deployment information, RSS modeling, and selection of an evaluation set of sample positions. This section corresponds to the first step shown in Fig. 2. Section III introduces fundamental lower bounds on the position estimation. This section corresponds to the second step shown in Fig. 2. Section IV introduces a general RSS threshold optimization procedure. This section corresponds to the third step shown in Fig. 2. In Section V, we validate the proposed framework experimentally with real RSS measurements. Finally, Section VI concludes the paper.

Throughout this paper, matrices are presented with uppercase letters and vectors with boldface lowercase letters. The operator $[\cdot]^{T}$ stands for vector/matrix transpose and $[\cdot]^{-1}$ stands for the inverse of a non-singular square matrix. The operator $\operatorname{tr}(\cdot)$ denotes the trace of a square matrix. $\|\cdot\|$ stands for the Euclidean norm of a vector and $|\cdot|$ denotes, depending on the context, either the cardinality of a set or the determinant of a matrix. The operator $\mathbb{E}(\cdot)$ stands for the statistical expectation. The operator $\ln (\cdot)$ stands for the natural $\operatorname{logarithm}$ and $\log (\cdot)$ stands for the logarithm to base 10 . Further, $\nabla_{\boldsymbol{\theta}}$ denotes the gradient operator. $\mathcal{N}\left(\mu, \sigma^{2}\right)$ denotes a Gaussian distribution with mean $\mu$ and variance $\sigma^{2}$. The operator $\operatorname{erf}(\cdot)$ stands for the standard Gaussian error function. Lastly, we use $\mathbf{I}_{N}, \mathbf{1}$ and $\mathbf{0}$ to denote an identity matrix of size $N \times N$, a vector of all $1 \mathrm{~s}$ and a vector of all 0 s, respectively.

The readers should not confuse the following notations: $\mathbf{p}$ geographical position, $\mathbf{P}$ a collection of geographical positions, $\overline{\mathbf{p}}$ grid position specific for the online Gaussian process regression, $\overline{\mathbf{P}}$ a set of grid positions, $\tilde{\mathbf{p}}$ test points for evaluating the Barankin bound, $P_{T}$ transmit power, $\mathbf{P}_{t h}$ a vector of RSS thresholds.

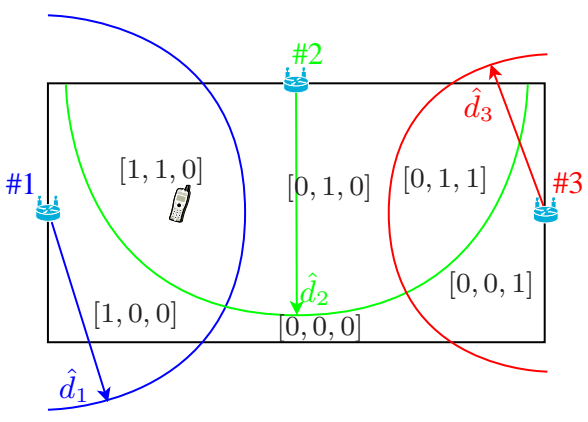

Fig. 1. Illustration of proximity report based indoor positioning. In this example, three reference nodes are deployed in a service area for positioning purposes. For simplicity, we assume that the RSS measurements are noise free and the underlying propagation model fits a linear log-distance model, cf. Section II-B. The coverage radius of each node, $\hat{d}_{i}, i=1,2,3$, is simply determined by $\hat{d}_{i}=d_{0} \cdot 10 \frac{P_{t h, i}-\hat{A}_{i}}{10 \hat{B}_{i}}$, where the notations will be explained later on. As we can see, the service area is divided into several small regions. An instantaneous RSS measurement being larger than a predefined threshold implies that the UE resides in the corresponding coverage area. For instance, the proximity vector, e.g., $[1,1,0]$, indicates that the UE is in the coverage area of the first and the second reference nodes, while outside of the coverage area of the third reference node. The UE needs to upload the proximity vector if and only if there is at least one entry reversing the status, for instance from $[1,1,0]$ to $[0,1,0]$, when the UE moved from the marked place some distance to the right.

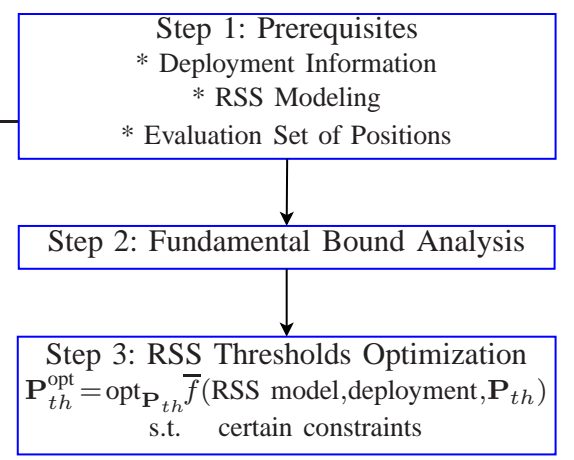

Fig. 2. Key steps of the proposed RSS thresholds optimization procedure. Herein, $\mathbf{P}_{t h}^{\text {opt }}$ represents the optimized RSS thresholds. The connections are the following. The fundamental bound analysis is based on deployment information and RSS modeling. The outcome of the fundamental bound analysis is the best achievable RMSE expression. Together with the evaluation set of positions, they are combined to evaluate the overall best achievable positioning accuracy as a function of the rss threshold.

\section{PREREQUisites}

Performing RSS threshold optimization in our work requires necessary deployment information, cf. Section II-A, a trained RSS model, cf. Section II-B, and an evaluation set of sample positions, cf. Section II-C.

\section{A. Deployment}

Throughout this paper, we restrict ourselves to indoor positioning scenarios where a number of $N$ reference network nodes, such as cellular base stations, BLE beacons, Wi-Fi routers, or a combination, are deployed. The reference network nodes are often placed rather uniformly in the surveillance area and mounted either on the ceiling or high on the wall to give a panoramic view. The geographical position and the transmission power of each reference network node are assumed to be 
known a priori. In addition to a reference signal, a reference network node may also broadcast information such as sensor ID, position, transmit power, network configurations, as well as a tiny amount of information about the event to be triggered.

\section{B. RSS Model}

A generic RSS model can be written as:

$$
r_{i}(\mathbf{p})=\underbrace{m_{i}(\mathbf{p})+e_{i}(\mathbf{p})}_{\bar{r}_{i}(\mathbf{p})}+n_{i}
$$

where $r_{i}(\mathbf{p})$ denotes the RSS observed at any position $\mathbf{p}$ (three dimensional in general) by the UE from the $i$ th reference network node, $m_{i}(\mathbf{p})$ denotes the received signal strength due to path loss as a deterministic function of $\mathbf{p}, e_{i}(\mathbf{p})$ is a position dependent noise term, and $n_{i}$ is a position independent noise term. Herein, we use $e_{i}(\mathbf{p})$ to represent the noise due to the shadowing effect, which is often position dependent. We use the noise term $n_{i}$ to account for the joint influence of the interference from other devices, signal absorption from human bodies, (unsuccessfully removed) small-scale fading, as well as the background noise. We assume $n_{i}$ to be independent and identically Gaussian distributed with zero mean and variance $\sigma_{n, i}^{2}$. The sum of $m_{i}(\mathbf{p})+e_{i}(\mathbf{p})$ denoted by $\bar{r}_{i}(\mathbf{p})$ can be understood as the clean RSS without noise. Model fitting can be done either offline or online. We show three examples in the sequel.

Example I: Offline Linear Least-Squares (LLS) Regression. The underlying received signal strength due to path loss is represented by a deterministic, linear log-distance model as follows:

$$
m_{i}(\mathbf{p})=A_{i}+10 B_{i} \log \left(\frac{d_{i}(\mathbf{p})}{d_{0}}\right),
$$

where $A_{i}$ is the received signal strength measured at a reference distance $d_{0}, B_{i}$ is the path loss exponent, and $d_{i}(\mathbf{p})$ is a short-hand notation of the Euclidean distance between $\mathbf{p}$ and the $i$ th reference network node's position, $\mathbf{p}_{\mathbf{r}, i}$, i.e., $d_{i}(\mathbf{p}) \triangleq\left\|\mathbf{p}-\mathbf{p}_{\mathbf{r}, i}\right\|$. Furthermore, we assume that the shadowing effect can be simply added to the variance $\sigma_{n, i}^{2}$ of $n_{i}$. The resulting RSS model can be seen as a special case of (1) and is widely known as log-distance path-loss model [10].

In the offline calibration phase, we are given a training data set, $\mathcal{D}_{i}=\left\{\left(\mathbf{p}_{i, j}, r_{i}\left(\mathbf{p}_{i, j}\right)\right) \mid j=1,2, \ldots, M\right\}$. In this example, we perform the well known offline LLS fitting and obtain an estimate of the unknown parameters, $\hat{\boldsymbol{\theta}}_{i}=\left[\hat{A}_{i}, \hat{B}_{i}, \hat{\sigma}_{n, i}^{2}\right]^{T}$. It is well known that $\hat{\boldsymbol{\theta}}_{i}$ can be obtained in closed form, see for instance [3]. Herein and in the subsequent examples, we use the calibrated parameters to replace the true parameter in the RSS prediction.

Finally, given a new position, $\mathbf{p}_{*}$, we can predict that the observed RSS measurement distributes as

$$
r_{i}\left(\mathbf{p}_{*}\right) \sim \mathcal{N}\left(\hat{\mu}_{i}\left(\mathbf{p}_{*}\right), \hat{\sigma}_{i}^{2}\left(\mathbf{p}_{*}\right)\right),
$$

where

$$
\begin{aligned}
& \hat{\mu}_{i}\left(\mathbf{p}_{*}\right)=m_{i}\left(\mathbf{p}_{*}\right)=A_{i}+10 B_{i} \log \left(\frac{d_{i}\left(\mathbf{p}_{*}\right)}{d_{0}}\right), \\
& \hat{\sigma}_{i}^{2}\left(\mathbf{p}_{*}\right)=\sigma_{n, i}^{2} .
\end{aligned}
$$

Example II: Offline Nonlinear Gaussian Process Regression (GPR). We follow the same path loss function as given in (2) in the first example. But the error term, $e_{i}(\mathbf{p})$, due to the large-scale shadow fading, is represented by a zero-mean Gaussian process

$$
e_{i}(\mathbf{p}) \sim \mathcal{G} \mathcal{P}\left(0, k_{i}\left(\mathbf{p}, \mathbf{p}^{\prime}\right)\right)
$$

where we follow the notation of a Gaussian process $\mathcal{G P}(\cdot, \cdot)$ used in [11, Section 2.2]. The covariance (kernel) function is selected to be

$$
k_{i}\left(\mathbf{p}, \mathbf{p}^{\prime}\right) \triangleq \mathbb{E}\left[e_{i}(\mathbf{p}) e_{i}\left(\mathbf{p}^{\prime}\right)\right]=\sigma_{s, i}^{2} \exp \left[\frac{-\left\|\mathbf{p}-\mathbf{p}^{\prime}\right\|}{l_{c, i}}\right],
$$

where $\sigma_{s, i}^{2}$ accounts for the uncertainty introduced by the shadow fading into the GP model and $l_{c, i}$ denotes the correlation distance. It is noted that in this model the noise terms at two different positions, say $\mathbf{p}$ and $\mathbf{p}^{\prime}$, are assumed to be spatially correlated according to the well-established Gudmundson's model [12]. Similar work but using different covariance functions can be found in [13]-[15].

Similar to the first example, we perform offline calibration of the GPR based RSS model. We start with writing the likelihood function of the observed RSS measurements as follows:

$$
p\left(\mathbf{r}_{i}\left(\mathbf{P}_{i}\right) ; \boldsymbol{\theta}_{i}\right) \sim \mathcal{N}\left(\mathbf{m}_{i}\left(\mathbf{P}_{i}\right), \mathbf{C}_{i}\left(\mathbf{P}_{i}, \mathbf{P}_{i}\right)\right),
$$

with the following notations:

$$
\begin{aligned}
& \boldsymbol{\theta}_{i} \triangleq\left[A_{i}, B_{i}, \sigma_{s, i}^{2}, l_{c, i}, \sigma_{n, i}^{2}\right]^{T}, \\
& \mathbf{P}_{i} \triangleq\left[\mathbf{p}_{i, 1}, \mathbf{p}_{i, 2}, \ldots, \mathbf{p}_{i, M}\right], \\
& \mathbf{r}_{i}\left(\mathbf{P}_{i}\right) \triangleq\left[r_{i}\left(\mathbf{p}_{i, 1}\right), r_{i}\left(\mathbf{p}_{i, 2}\right), \ldots, r_{i}\left(\mathbf{p}, \mathbf{p}_{i}\left(\mathbf{p}_{i, 2}\right), \ldots,\right.\right. \\
& \mathbf{m}_{i}\left(\mathbf{P}_{i}\right) \triangleq\left[m_{i}\left(\mathbf{p}_{i, 1}\right), m_{i},\right. \triangleq\left[k_{i}\left(\mathbf{p}, \mathbf{p}_{i, 1}\right), k_{i}\left(\mathbf{p}, \mathbf{p}_{i, 2}\right), .\right. \\
& \mathbf{k}_{i}\left(\mathbf{p}, \mathbf{P}_{i}\right) \\
& \mathbf{K}_{i}\left(\mathbf{P}_{i}, \mathbf{P}_{i}\right) \triangleq\left[\begin{array}{c}
\left.\mathbf{k}_{i}\left(\mathbf{p}_{i, 1}, \mathbf{P}_{i}\right)\right] \\
\mathbf{k}_{i}\left(\mathbf{p}_{i, 2}, \mathbf{P}_{i}\right) \\
\vdots \\
\mathbf{k}_{i}\left(\mathbf{p}_{i, M}, \mathbf{P}_{i}\right)
\end{array}\right], \\
& \mathbf{C}_{i}\left(\mathbf{P}_{i}, \mathbf{P}_{i}\right) \triangleq \mathbf{K}_{i}\left(\mathbf{P}_{i}, \mathbf{P}_{i}\right)+\sigma_{n, i}^{2} \mathbf{I}_{M} .
\end{aligned}
$$$$
\mathbf{r}_{i}\left(\mathbf{P}_{i}\right) \triangleq\left[r_{i}\left(\mathbf{p}_{i, 1}\right), r_{i}\left(\mathbf{p}_{i, 2}\right), \ldots, r_{i}\left(\mathbf{p}_{i, M}\right)\right]^{T},
$$$$
\mathbf{m}_{i}\left(\mathbf{P}_{i}\right) \triangleq\left[m_{i}\left(\mathbf{p}_{i, 1}\right), m_{i}\left(\mathbf{p}_{i, 2}\right), \ldots, m_{i}\left(\mathbf{p}_{i, M}\right)\right]^{T},
$$$$
\mathbf{k}_{i}\left(\mathbf{p}, \mathbf{P}_{i}\right) \triangleq\left[k_{i}\left(\mathbf{p}, \mathbf{p}_{i, 1}\right), k_{i}\left(\mathbf{p}, \mathbf{p}_{i, 2}\right), \ldots, k_{i}\left(\mathbf{p}, \mathbf{p}_{i, M}\right)\right]^{T},
$$

The parameters in $\boldsymbol{\theta}_{i}$ are usually unknown and need to be calibrated. A maximum-likelihood estimate (MLE), $\hat{\boldsymbol{\theta}}_{i}$, is adopted here as an approximation of the underlying parameters. Detailed derivations are given in Appendix A.

In order to give a training data driven RSS model that takes into account all noise sources, we compute according to [11] the Gaussian posterior probability of an observed RSS value at a new position $\mathbf{p}_{*}$ by

$$
p\left(r_{i}\left(\mathbf{p}_{*}\right) \mid \mathcal{D}_{i} ; \hat{\boldsymbol{\theta}}_{i}\right) \sim \mathcal{N}\left(\hat{\mu}_{i}\left(\mathbf{p}_{*}\right), \hat{\sigma}_{i}^{2}\left(\mathbf{p}_{*}\right)\right),
$$

where

$$
\begin{gathered}
\hat{\mu}_{i}\left(\mathbf{p}_{*}\right)=\mathbf{k}_{i}^{T}\left(\mathbf{p}_{*}\right) \mathbf{C}_{i}^{-1}\left(\mathbf{r}_{i}-\mathbf{m}_{i}\right)+m_{i}\left(\mathbf{p}_{*}\right) \\
\hat{\sigma}_{i}^{2}\left(\mathbf{p}_{*}\right)=\sigma_{n, i}^{2}+\sigma_{s, i}^{2}-\mathbf{k}_{i}^{T}\left(\mathbf{p}_{*}\right) \mathbf{C}_{i}^{-1} \mathbf{k}_{i}\left(\mathbf{p}_{*}\right) .
\end{gathered}
$$

Note that in (10a) and (10b), $\mathbf{r}_{i}, \mathbf{m}_{i}, \mathbf{k}_{i}\left(\mathbf{p}_{*}\right)$, and $\mathbf{C}_{i}$ are short for $\mathbf{r}_{i}\left(\mathbf{P}_{i}\right), \mathbf{m}_{i}\left(\mathbf{P}_{i}\right), \mathbf{k}_{i}\left(\mathbf{p}_{*}, \mathbf{P}_{i}\right)$, and $\mathbf{C}_{i}\left(\mathbf{P}_{i}, \mathbf{P}_{i}\right)$. 
The above introduced GPR will be called full GPR in the sequel for the reason that it processes the training data (of size $M$ ) in a batch manner. The corresponding computational complexity scales as $\mathcal{O}\left(M^{3}\right)$ and the memory requirement scales as $\mathcal{O}\left(M^{2}\right)$. Some sparse GPR methods have been proposed to reduce the computational complexity to $\mathcal{O}\left(s^{2} M\right)$ and the memory requirement to $\mathcal{O}(s M)$, where $s(s \ll M)$ is the size of a set of inducing input points that are sub-sampled smartly from the original training dataset. A unifying view of the state-of-the-art sparse GPR methods has been given in [16]. Next, we apply the recent recursive Gaussian process regression method [17] for deriving our online RSS model.

Example III: Online Gaussian Process Regression. The notations, if not re-defined, will follow those given for the full GPR method. For simplicity and easier comparison with the full GPR, let us imagine that the training data arrives one by one as time evolves, namely we have a new data point $\left\{\mathbf{p}_{i, t}, r_{i}\left(\mathbf{p}_{i, t}\right)\right\}$, at each time instance $t=1,2, \ldots, M$.

Similar to the sparse GPR methods, we introduce a set of $s$ grids $\overline{\mathbf{P}}=\left[\overline{\mathbf{p}}_{1}, \overline{\mathbf{p}}_{2}, \ldots, \overline{\mathbf{p}}_{s}\right]$, representing the desired tracking area. Herein, we simply assume that all the reference nodes use the same $\overline{\mathbf{P}}$. The corresponding RSS observations at these grids are latent variables $\overline{\mathbf{r}}_{i}(\overline{\mathbf{P}}) \triangleq$ $\left[\bar{r}_{i}\left(\overline{\mathbf{p}}_{1}\right), \bar{r}_{i}\left(\overline{\mathbf{p}}_{2}\right), \ldots, \bar{r}_{i}\left(\overline{\mathbf{p}}_{s}\right)\right]^{T}$. Furthermore, we denote $\mathcal{D}_{g, i} \triangleq$ $\left\{\overline{\mathbf{r}}_{i}(\overline{\mathbf{P}}), \overline{\mathbf{P}}\right\}$. For notational brevity in the sequel, $\overline{\mathbf{r}}_{i}$ is short for $\overline{\mathbf{r}}_{i}(\overline{\mathbf{P}})$, and its mean and covariance matrix are denoted by $\overline{\mathbf{m}}_{i}$ and $\overline{\mathbf{K}}_{i}$, respectively. Our first aim is to compute the posterior distribution of $\overline{\mathbf{r}}_{i}$ at any time instance $t(t \geq 1)$ given the training data $\mathcal{D}_{i, 1: t} \triangleq\left\{\mathbf{P}_{i, 1: t}, \mathbf{r}_{i}\left(\mathbf{P}_{i, 1: t}\right)\right\}$. Herein, the noisy RSS observations are stacked into $\mathbf{r}_{i}\left(\mathbf{P}_{i, 1: t}\right) \triangleq$ $\left[r_{i}\left(\mathbf{p}_{i, 1}\right), r_{i}\left(\mathbf{p}_{i, 2}\right), \ldots, r_{i}\left(\mathbf{p}_{i, t}\right)\right]^{T}$, and the locations are stacked into $\mathbf{P}_{i, 1: t} \triangleq\left[\mathbf{p}_{i, 1}, \mathbf{p}_{i, 2}, \ldots, \mathbf{p}_{i, t}\right]$.

The main steps of the recursive GPR [17] based RSS model are summarized as follows:

1) Initialization: Set initial mean vector $\boldsymbol{\mu}_{i, 0}^{g} \triangleq \overline{\mathbf{m}}_{i}$ and the covariance matrix $\mathbf{C}_{i, 0}^{g} \triangleq \overline{\mathbf{K}}_{i}$. Compute the inverse of $\overline{\mathbf{K}}_{i}$ and store it for later use.

2) Recursive Processing: For each $t=1,2, \ldots, M$, do the following computations:

$$
\begin{aligned}
\mathbf{J}_{i, t} & =\mathbf{k}_{i}\left(\mathbf{p}_{i, t}, \overline{\mathbf{P}}\right) \overline{\mathbf{K}}_{i}^{-1}, \\
\mu_{i, t}^{p} & =m_{i}\left(\mathbf{p}_{i, t}\right)+\mathbf{J}_{i, t}\left(\boldsymbol{\mu}_{i, t-1}^{g}-\overline{\mathbf{m}}_{i}\right), \\
\sigma_{i, t}^{2, p} & =\sigma_{s, i}^{2}+\mathbf{J}_{i, t}\left(\mathbf{C}_{i, t-1}^{g}-\overline{\mathbf{K}}_{i}\right) \mathbf{J}_{i, t}^{T}, \\
\tilde{\mathbf{G}}_{i, t} & =\frac{1}{\sigma_{n, i}^{2}+\left(\sigma_{i, t}^{p}\right)^{2}} \mathbf{C}_{i, t-1}^{g} \mathbf{J}_{i, t}^{T}, \\
\boldsymbol{\mu}_{i, t}^{g} & =\boldsymbol{\mu}_{i, t-1}^{g}+\tilde{\mathbf{G}}_{i, t}\left(r_{i}\left(\mathbf{p}_{i, t}\right)-\mu_{i, t}^{p}\right), \\
\mathbf{C}_{i, t}^{g} & =\mathbf{C}_{i, t-1}^{g}-\tilde{\mathbf{G}}_{i, t} \mathbf{J}_{i, t} \mathbf{C}_{i, t-1}^{g} .
\end{aligned}
$$

After the recursive processing of the online GPR, cf. (11a)-(11f), we have

$$
p\left(\overline{\mathbf{r}}_{i} \mid \overline{\mathbf{P}}, \mathcal{D}_{i}\right) \sim \mathcal{N}\left(\boldsymbol{\mu}_{i, M}^{g}, \mathbf{C}_{i, M}^{g}\right) .
$$

3) Prediction: At the end of the training phase, i.e., $t=M$ assumed in this specific example, the posterior distribution of $r_{i}\left(\mathbf{p}_{*}\right)$ at a novel input position $\mathbf{p}_{*}$, given $\mathcal{D}_{i}$ and $\mathcal{D}_{g, i}$, can be approximated by

$$
p\left(r_{i}\left(\mathbf{p}_{*}\right) \mid \mathbf{p}_{*}, \overline{\mathbf{P}}, \mathcal{D}_{i}\right) \approx \mathcal{N}\left(r_{i}\left(\mathbf{p}_{*}\right) \mid \hat{\mu}_{i}\left(\mathbf{p}_{*}\right), \hat{\sigma}_{i}^{2}\left(\mathbf{p}_{*}\right)\right),
$$

where

$$
\begin{gathered}
\hat{\mu}_{i}\left(\mathbf{p}_{*}\right)=\overline{\mathbf{k}}_{i}^{T}\left(\mathbf{p}_{*}\right) \overline{\mathbf{K}}_{i}^{-1}\left(\boldsymbol{\mu}_{i, M}^{g}-\overline{\mathbf{m}}_{i}\right)+m_{i}\left(\mathbf{p}_{*}\right), \quad(14 \mathrm{a}) \\
\hat{\sigma}_{i}^{2}\left(\mathbf{p}_{*}\right)=\sigma_{s, i}^{2}+\sigma_{n, i}^{2}+\overline{\mathbf{k}}_{i}^{T}\left(\mathbf{p}_{*}\right) \overline{\mathbf{K}}_{i}^{-1}\left(\mathbf{C}_{i, M}^{g} \overline{\mathbf{K}}_{i}^{-1}-\mathbf{I}_{s}\right) \overline{\mathbf{k}}_{i}\left(\mathbf{p}_{*}\right) .
\end{gathered}
$$

Herein, $\overline{\mathbf{k}}_{i}\left(\mathbf{p}_{*}\right)$ is short for $\mathbf{k}_{i}\left(\mathbf{p}_{*}, \overline{\mathbf{P}}\right)$.

Note that the detailed derivations of (11a)-(11f) can be found in [17] and the derivations of (14a) and (14b) are given in Appendix B. It is easy to verify that the computational complexity scales as $\mathcal{O}\left(s^{3}\right)$ for evaluating $\overline{\mathbf{K}}_{i}^{-1}$ only once in the initialization step, $\mathcal{O}\left(s^{2}\right)$ for $\boldsymbol{\mu}_{i, t}^{g}$ and $\mathbf{C}_{i, t}^{g}$ at any time instance $t$ in the recursive processing step. As compared to the full GPR method, online GPR method is able to reduce the overall computational complexity from $\mathcal{O}\left(M^{3}\right)$ to $\mathcal{O}\left(s^{2} M\right)$ with $s \ll M$. Although the online GPR method provides the same overall computational complexity as some sparse GPR methods, it can process new data online with less computational effort. More precisely, when we have a new input/observation pair $\left\{\mathbf{p}_{i, M+1}, r_{i}\left(\mathbf{p}_{i, M+1}\right)\right\}$ at time $M+1$ after the training phase, it requires only $\mathcal{O}\left(s^{2}\right)$ complexity to compute $\boldsymbol{\mu}_{i, M+1}^{g}$ and $\mathbf{C}_{i, M+1}^{g}$.

In the above RSS modeling procedure, we assumed that an estimate of the true parameter $\boldsymbol{\theta}_{i}$ is known prior to the online GPR. This can be the case when some historical/expert knowledge is available or a small set of the training data can be used to train the parameters like we did for the full GP in the second example. Alternatively, [18] demonstrated that these parameters can be learned online as training data come in. To summarize, online GPR model is more flexible to use and adaptive to new arrival data. While if the underlying RSS model is time invariant and the computational cost is secondary, full GPR using all available measurements for both hyper-parameter optimization and prediction will theoretically give the best modeling results.

\section{Evaluation Set of Sample Positions}

Apart from the deployment information and the RSS model, we need also an evaluation set of sample positions for which localization accuracy will be evaluated. An evaluation set can be selected for instance to contain uniform grids or a plurality of trajectories that cover the area where positioning is of interest.

\section{Concluding Remarks}

This section introduces and compares several RSS models. A good RSS model is able to give more information about the UE position in a proximity measurements, which is essentially a hard-thresholded RSS measurement. The Gaussian distributive profile of the RSS makes the computation of an information metric tractable. More details will be given at beginning of Section III. 


\section{Fundamental Lower Bounds on Position ESTIMATION}

This section aims to derive two fundamental lower bounds on the proximity report based position estimation meansquare-error (MSE). These are the Cramér-Rao bound (CRB) and the Barankin bound (BB), respectively. It is known that $\mathrm{CRB}$ falls into the category of small error bounds, while $\mathrm{BB}$ falls into the category of large error bounds. Small/Large-error bound is intended for small/large estimation error [19]. Herein, we assume that all regularity conditions for computing these two bounds are fulfilled.

\section{A. Preliminaries}

A proximity report is obtained by hard thresholding an RSS measurement, concretely,

$$
c_{i}=\left\{\begin{array}{ll}
0, & r_{i}(\mathbf{p}) \leq P_{t h, i} \\
1, & r_{i}(\mathbf{p})>P_{t h, i}
\end{array},\right.
$$

where $c_{i}$ is introduced here to denote the proximity information obtained through comparing a threshold $P_{t h, i}$ with the instantaneous RSS value, $r_{i}$, measured at $\mathbf{p}=[x, y, z]^{T}$ by the UE via communicating with the $i$ th reference network node. We set $c_{i}$ equal to ' 1 '/'0'when the UE is in/beyond proximity of the $i$ th reference network node. We further define a vector of RSS measurements received by the UE from all reference network nodes as $\mathbf{r}=\left[r_{1}, r_{2}, \ldots, r_{N}\right]^{T}$ and define the corresponding vector of proximity reports as $\mathbf{c}=\left[c_{1}, c_{2}, \ldots, c_{N}\right]^{T}$.

By taking advantages of the Gaussian distributive profile of (3) for the log-distance model or (9) for the full GPR model or (13) for the online GPR model, we can easily derive the probability of observing $c_{i}$ at $\mathbf{p}$, i.e.,

$$
\operatorname{Pr}\left\{c_{i} ; \mathbf{p}\right\}=\left\{\begin{array}{ll}
G\left(\frac{P_{t h, i}-\hat{\mu}_{i}(\mathbf{p})}{\hat{\sigma}_{i}(\mathbf{p})}\right), & c_{i}=0 \\
1-G\left(\frac{P_{t h, i}-\hat{\mu}_{i}(\mathbf{p})}{\hat{\sigma}_{i}(\mathbf{p})}\right), & c_{i}=1
\end{array},\right.
$$

where $G(\cdot)$ denotes the Gaussian cumulative distribution function $(\mathrm{CDF})$. For notational brevity, we define $G_{i}(\mathbf{p}) \triangleq$ $G\left(\frac{P_{t h, i}-\hat{\mu}_{i}(\mathbf{p})}{\hat{\sigma}_{i}(\mathbf{p})}\right)$. Similarly, we use $\operatorname{Pr}\{\mathbf{c} ; \mathbf{p}\}$ to denote the probability of observing vector $\mathbf{c}$ at $\mathbf{p}$ and due to the assumption that the measurements are mutually independent, we have $\operatorname{Pr}\{\mathbf{c} ; \mathbf{p}\}=\prod_{i=1}^{N} \operatorname{Pr}\left\{c_{i} ; \mathbf{p}\right\}$. Lastly, we note that in $(16) \hat{\mu}_{i}(\mathbf{p})$ and $\hat{\sigma}_{i}(\mathbf{p})$ follow the expressions given in (4a) and (4b) for the linear log-distance model, or (10a) and (10b) for the full GPR model, or (14a) and (14b) for the online GPR model.

\section{B. Proximity Report Based Position Estimator}

We define a proximity based position estimator by $\hat{\mathbf{p}}(\mathbf{c})$. A direct measure of the estimation error-the mean-square-error (MSE), is very often used for comparing different parameter estimators. The MSE of any proximity based position estimator can be expressed as

$$
\begin{aligned}
\operatorname{MSE}(\hat{\mathbf{p}}(\mathbf{c})) & \triangleq \mathbb{E}_{\operatorname{Pr}\{\mathbf{c} ; \mathbf{p}\}}\left[(\hat{\mathbf{p}}(\mathbf{c})-\mathbf{p})(\hat{\mathbf{p}}(\mathbf{c})-\mathbf{p})^{T}\right] \\
& =\mathbf{b}(\mathbf{p}) \mathbf{b}^{T}(\mathbf{p})+\Sigma(\mathbf{p}),
\end{aligned}
$$

where the bias, $\mathbf{b}(\mathbf{p})$, and the covariance matrix, $\Sigma(\mathbf{p})$, are defined, respectively, by

$$
\begin{aligned}
& \mathbf{b}(\mathbf{p}) \triangleq \mathbb{E}_{\operatorname{Pr}\{\mathbf{c} ; \mathbf{p}\}}[\hat{\mathbf{p}}(\mathbf{c})]-\mathbf{p}, \\
& \boldsymbol{\rho}(\mathbf{p}) \triangleq \mathbb{E}_{\operatorname{Pr}\{\mathbf{c} ; \mathbf{p}\}}\{\hat{\mathbf{p}}(\mathbf{c})]=\mathbf{b}(\mathbf{p})+\mathbf{p}, \\
& \Sigma(\mathbf{p}) \triangleq \mathbb{E}_{\operatorname{Pr}\{\mathbf{c} ; \mathbf{p}\}}\left[(\hat{\mathbf{p}}(\mathbf{c})-\boldsymbol{\rho}(\mathbf{p}))(\hat{\mathbf{p}}(\mathbf{c})-\boldsymbol{\rho}(\mathbf{p}))^{T}\right] .
\end{aligned}
$$

\section{Fundamental Lower Bounds}

It is known from the literature, see for instance [19], that fundamental lower bounds can be placed on the MSE of a parameter estimator by

$$
\operatorname{MSE}(\hat{\mathbf{p}}(\mathbf{c})) \succeq \mathbf{b}(\mathbf{p}) \mathbf{b}^{T}(\mathbf{p})+\Upsilon(\mathbf{p}) \Gamma(\mathbf{p})^{-1} \Upsilon(\mathbf{p})^{T},
$$

where the information matrix, $\Gamma(\mathbf{p})$, and the translation matrix, $\Upsilon(\mathbf{p})$, are defined as follows:

$$
\begin{aligned}
& \Gamma(\mathbf{p}) \triangleq \mathbb{E}_{\operatorname{Pr}\{\mathbf{c} ; \mathbf{p}\}}\left[\boldsymbol{\eta}(\mathbf{p}) \boldsymbol{\eta}(\mathbf{p})^{T}\right], \\
& \Upsilon(\mathbf{p}) \triangleq \mathbb{E}_{\operatorname{Pr}\{\mathbf{c} ; \mathbf{p}\}}\left[(\hat{\mathbf{p}}(\mathbf{c})-\boldsymbol{\rho}(\mathbf{p})) \boldsymbol{\eta}(\mathbf{p})^{T}\right],
\end{aligned}
$$

where $\boldsymbol{\eta}(\mathbf{p})$ is some function of $\mathbf{p}$. Next, we show two well known lower bounds that adopt different $\boldsymbol{\eta}(\mathbf{p})$.

Cramér-Rao Bound (CRB): When we let

$$
\boldsymbol{\eta}(\mathbf{p})=\frac{\frac{\partial}{\partial \mathbf{p}} \operatorname{Pr}\{\mathbf{c} ; \mathbf{p}\}}{\operatorname{Pr}\{\mathbf{c} ; \mathbf{p}\}}
$$

the translation matrix, $\Upsilon(\mathbf{p})$, defined in (21) can be easily proven to become

$$
\Upsilon(\mathbf{p})=\left(\frac{\partial \boldsymbol{\rho}^{T}(\mathbf{p})}{\partial \mathbf{p}}\right)^{T}
$$

and the information matrix, $\Gamma(\mathbf{p})$, defined in (20) becomes the well known Fisher information matrix (FIM), namely,

$$
\Gamma(\mathbf{p})=\mathbb{E}_{\operatorname{Pr}\{\mathbf{c} ; \mathbf{p}\}}\left[\frac{\frac{\partial}{\partial \mathbf{p}} \operatorname{Pr}\{\mathbf{c} ; \mathbf{p}\}\left(\frac{\partial}{\partial \mathbf{p}} \operatorname{Pr}\{\mathbf{c} ; \mathbf{p}\}\right)^{T}}{\operatorname{Pr}^{2}\{\mathbf{c} ; \mathbf{p}\}}\right] .
$$

The FIM can be further expressed, due to the independence assumption on the measurements, as

$$
\begin{aligned}
\Gamma(\mathbf{p}) & =\sum_{i=1}^{N} \mathbb{E}_{\operatorname{Pr}\left\{c_{i} ; \mathbf{p}\right\}}\left[\frac{\frac{\partial}{\partial \mathbf{p}} \operatorname{Pr}\left\{c_{i} ; \mathbf{p}\right\}\left(\frac{\partial}{\partial \mathbf{p}} \operatorname{Pr}\left\{c_{i} ; \mathbf{p}\right\}\right)^{T}}{\operatorname{Pr}^{2}\left\{c_{i} ; \mathbf{p}\right\}}\right] \\
& \triangleq \sum_{i=1}^{N}\left[\begin{array}{lll}
f_{i, x x} & f_{i, x y} & f_{i, x z} \\
f_{i, y x} & f_{i, y y} & f_{i, y z} \\
f_{i, z x} & f_{i, z y} & f_{i, z z}
\end{array}\right],
\end{aligned}
$$

where for any combination of $m, n \in\{x, y, z\}$,

$$
\begin{aligned}
f_{i, m n} & =\sum_{c_{i} \in\{0,1\}} \frac{\frac{\partial}{\partial m} \operatorname{Pr}\left\{c_{i} ; \mathbf{p}\right\} \cdot \frac{\partial}{\partial n} \operatorname{Pr}\left\{c_{i} ; \mathbf{p}\right\}}{\operatorname{Pr}\left\{c_{i} ; \mathbf{p}\right\}} \\
& =\left(\frac{\partial G_{i}(\mathbf{p})}{\partial m} \cdot \frac{\partial G_{i}(\mathbf{p})}{\partial n}\right) \cdot\left(\frac{1}{G_{i}(\mathbf{p})}+\frac{1}{1-G_{i}(\mathbf{p})}\right) .
\end{aligned}
$$

The first-order derivatives of $G_{i}(\mathbf{p})$ for all the three RSS models are given in Appendix C. Having $G_{i}(\mathbf{p})$ as well as its derivatives, we can easily evaluate (25) to get the FIM. 
Finally, inserting the results of $\Upsilon(\mathbf{p})$ and $\Gamma(\mathbf{p})$ into the righthand-side of (19) gives the CRB.

Barankin Bound (BB): In this case, as will be shown later on, $\boldsymbol{\eta}(\mathbf{p})$ and eventually $\Upsilon(\mathbf{p})$ and $\Gamma(\mathbf{p})$ are also functions of an additional set of test points. This set of test points, denoted as $\tilde{\mathbf{p}}_{j}, j=1,2, \ldots, M_{b}$, must be preselected according to a given UE position $\mathbf{p}$. This set of test points decides the tightness of the Barankin bound to be derived below.

For the BB, we set

$$
\boldsymbol{\eta}(\mathbf{p})=\left[\frac{\operatorname{Pr}\left\{\mathbf{c} ; \tilde{\mathbf{p}}_{1}\right\}}{\operatorname{Pr}\{\mathbf{c} ; \mathbf{p}\}}, \frac{\operatorname{Pr}\left\{\mathbf{c} ; \tilde{\mathbf{p}}_{2}\right\}}{\operatorname{Pr}\{\mathbf{c} ; \mathbf{p}\}}, \ldots, \frac{\operatorname{Pr}\left\{\mathbf{c} ; \tilde{\mathbf{p}}_{M_{b}}\right\}}{\operatorname{Pr}\{\mathbf{c} ; \mathbf{p}\}}\right]^{T}-\mathbf{1}^{T}
$$

The translation matrix can be derived based on the results in [19] to be

$$
\Upsilon(\mathbf{p})=\left[\boldsymbol{\rho}\left(\tilde{\mathbf{p}}_{1}\right)-\boldsymbol{\rho}(\mathbf{p}), \boldsymbol{\rho}\left(\tilde{\mathbf{p}}_{2}\right)-\boldsymbol{\rho}(\mathbf{p}), \ldots, \boldsymbol{\rho}\left(\tilde{\mathbf{p}}_{M_{b}}\right)-\boldsymbol{\rho}(\mathbf{p})\right],
$$

and the information matrix $\Gamma(\mathbf{p})$ becomes the Barankin information matrix (of dimension $M_{b} \times M_{b}$ ), with the $(j, k)$ th entry given by

$$
\begin{aligned}
\Gamma_{j k}\left(\mathbf{p}, \tilde{\mathbf{p}}_{j}, \tilde{\mathbf{p}}_{k}\right) & =\mathbb{E}_{\operatorname{Pr}\{\mathbf{c} ; \mathbf{p}\}}\left[\frac{\operatorname{Pr}\left\{\mathbf{c} ; \tilde{\mathbf{p}}_{j}\right\}}{\operatorname{Pr}\{\mathbf{c} ; \mathbf{p}\}} \frac{\operatorname{Pr}\left\{\mathbf{c} ; \tilde{\mathbf{p}}_{k}\right\}}{\operatorname{Pr}\{\mathbf{c} ; \mathbf{p}\}}\right]-1, \quad(29) \\
& =\prod_{i=1}^{N}\left(\sum_{c_{i} \in\{0,1\}} \frac{\operatorname{Pr}\left\{c_{i} ; \tilde{\mathbf{p}}_{j}\right\} \operatorname{Pr}\left\{c_{i} ; \tilde{\mathbf{p}}_{k}\right\}}{\operatorname{Pr}\left\{c_{i} ; \mathbf{p}\right\}}\right)-1,
\end{aligned}
$$

where the second equation is owing to the independence assumption on the proximity reports.

In order to give a tight lower bound, the test points need to be carefully selected. The optimal set of test points can be found as

$$
\left[\tilde{\mathbf{p}}_{1}^{\mathrm{opt}}, \tilde{\mathbf{p}}_{2}^{\mathrm{opt}}, \ldots, \tilde{\mathbf{p}}_{M_{b}}^{\mathrm{opt}}\right]=\max _{\tilde{\mathbf{p}}_{1}, \ldots, \tilde{\mathbf{p}}_{M_{b}}} \Upsilon(\mathbf{p}) \Gamma(\mathbf{p})^{-1} \Upsilon(\mathbf{p})^{T} .
$$

Unfortunately, this optimization problem is cumbersome in our case. A much easier way of providing a set of sub-optimal test points is to minimize the diagonal elements of the Barankin matrix, $\Gamma_{j j}\left(\mathbf{p}, \tilde{\mathbf{p}}_{j}\right)$, namely,

$$
\tilde{\mathbf{p}}_{j}^{\text {s-opt }}=\arg \min _{\tilde{\mathbf{p}}_{j}} \Gamma_{j j}\left(\mathbf{p}, \tilde{\mathbf{p}}_{j}\right), \quad \forall j=1,2, \ldots, M_{b} .
$$

According to (30), we have

$$
\Gamma_{j j}\left(\mathbf{p}, \tilde{\mathbf{p}}_{j}\right)=\prod_{i=1}^{N}\left(\sum_{c_{i} \in\{0,1\}} \frac{\operatorname{Pr}^{2}\left\{c_{i} ; \tilde{\mathbf{p}}_{j}\right\}}{\operatorname{Pr}\left\{c_{i} ; \mathbf{p}\right\}}\right)-1 .
$$

We adopt the following procedure to find the test points:

1) Generate a set of $K$ (with $K \gg M_{b}$ ) candidate test points, $\tilde{\mathbf{p}}_{k}$, uniformly and rather densely in the service area.

2) For each candidate test point, evaluate $\Gamma_{k k}, k=$ $1,2, \ldots, K$, according to (33) and store them.

3) Find among $\Gamma_{k k}, k=1,2, \ldots, K$, all the local minima $\Gamma_{j^{\prime} j^{\prime}}$ and record their indexes $j^{\prime}$.

4) Set a selection threshold, $\varepsilon$, search for all $j \in\left\{1, \ldots, j^{\prime}-\right.$ $\left.1, j^{\prime}+1, \ldots, K\right\}$ that satisfy $\Gamma_{j j}-\Gamma_{j^{\prime} j^{\prime}}<\varepsilon$ and use $\tilde{\mathbf{p}}_{j}$ as the final test points. Repeat this for all $j^{\prime}$.
The test points selected in the above way should well represent the location ambiguities that a position estimation algorithm gets stuck at more easily. Another big advantage of the BB lies in that the map information can be easily incorporated into the test point selection procedure. Lastly, we take the optimized test points $\tilde{\mathbf{p}}_{j}^{\text {s-opt }}, j=1,2, \ldots, M_{b}$ into (28) and (30) to evaluate $\Upsilon(\mathbf{p})$ and $\Gamma(\mathbf{p})$, respectively. Similarly, inserting the results of $\Upsilon(\mathbf{p})$ and $\Gamma(\mathbf{p})$ into the right-hand-side of (19) gives the BB. It was shown in [20, Theorem I] that the Barankin bound is an increasing function of the number of test points and can converge to certain value when the regularity conditions are fulfilled. We have confirmed this experimentally in [2].

\section{Computational Complexity}

Before showing the results, let us first recap the following notations: $N$ is the number of reference network nodes, $M$ the number of RSS measurements collected at each node, $s$ the number of grids for the online GP regression, $K$ the total number of candidate test points and $M_{b}$ the final candidate test points for computing the Barankin bound.

The computation of the CRB requires the first order derivatives of $G_{i}(\mathbf{p})$. From our derivations in Appendix C, the computational complexity scales as $\mathcal{O}(N)$ for the linear RSS model, while scales as $\mathcal{O}\left(N M^{2}\right)$ for the full GPR model and scales as $\mathcal{O}\left(N s^{2}\right)$ for the online GPR model. The computation of the $\mathrm{BB}$ does not require the first order derivatives of $G_{i}(\mathbf{p})$ as in the CRB computation, but it requires a set of carefully selected test points. It is easy to derive from the above subsection that the computational complexity scales as $\mathcal{O}(N K)+\mathcal{O}\left(M_{b}^{3}\right)$. One practical way to suppress the high complexity is to make $K$ and $M_{b}$ relatively small at the cost of reduced accuracy of the Barankin bound.

\section{E. Discussions on Bias}

In the previous subsection, we derived two fundamental lower bounds, namely the $\mathrm{CRB}$ and the $\mathrm{BB}$, on the MSE of any proximity based position estimator. Obviously, it is much simpler to compute the two bounds for unbiased position estimator due to the fact that $\mathbf{b}(\mathbf{p})=\mathbf{0}, \forall \mathbf{p}$ in the parameter space. Two different methods might be used to derive an unbiased, proximity report based position estimator. In the first method, an unbiased estimator might be obtained as a by-product when deriving the $\mathrm{BB}$ in a more complicated manner according to [21]. In the second method, we might use dithering techniques to generate dither noise and add it to the proximity measurement before running the maximumlikelihood estimation. We have shown some interesting results recently in [22], [23]. In the sequel, we will focus on unbiased position estimator. However, our method also applies for any biased estimator if the bias $\mathbf{b}(\mathbf{p})$ is of known form, which needs to be obtained either analytically or replaced by the maximum bias tolerance planned for each position in the worst case.

\section{RSS THRESHOLD OPTIMIZATION}

In Section II and Section III, we listed the prerequisites and information criteria that serve as elementary building blocks 
for RSS threshold optimization. In this section, we aim to give a generic RSS thresholding procedure, followed by discussions on the implementation aspect.

We summarize the results obtained from Section II as follows:

- We obtained the sensor deployment information, including for instance the floor plan, reference network node positions, transmit power, etc.

- We obtained a calibrated RSS model for each reference network node $i, i=1,2, \ldots, N$, specified by the model parameters:

1) $\hat{\boldsymbol{\theta}}_{i}=\left[\hat{A}_{i}, \hat{B}_{i}, \hat{\sigma}_{i}^{2}\right]^{T}$ in the linear log-distance model, cf. Example I given in Section II-B; or

2) $\hat{\boldsymbol{\theta}}_{i}=\left[\hat{A}_{i}, \hat{B}_{i}, \hat{\sigma}_{s, i}^{2}, \hat{l}_{c, i}, \hat{\sigma}_{n, i}^{2}\right]$ in the nonlinear GPR model, cf. Example II and Example III given in Section II-B.

- We obtained an evaluation set, $\mathcal{X}^{*}$, which contains known sample positions $\mathbf{p}_{i}^{*} \triangleq\left[x_{i}^{*}, y_{i}^{*}, z_{i}^{*}\right]^{T}$ that might be considered to be of varied importance quantified by the weighting factors $w_{i}^{*}, i=1,2, \ldots,\left|\mathcal{X}^{*}\right|$. The weighting factors are positive and sum up to one.

Next, we tune the thresholds $\mathbf{P}_{t h}=\left[P_{t h, 1}, \ldots, P_{t h, N}\right]$ of the $N$ reference nodes. The workings are as follows:

1) For every sample position $\mathbf{p}_{i}^{*} \in \mathcal{X}^{*}$, evaluate some localization accuracy metric $f\left(\mathbf{p}_{i}^{*}, \mathbf{P}_{t h}\right)$. Take weighted average by

$$
\bar{f}\left(\mathbf{P}_{t h}\right)=\sum_{i=1}^{\left|\mathcal{X}^{*}\right|} w_{i}^{*} f\left(\mathbf{p}_{i}^{*}, \mathbf{P}_{t h}\right),
$$

where $\bar{f}\left(\mathbf{P}_{t h}\right)$ is short for an overall accuracy metric function that is dependent of deployment and RSS model, and $f\left(\mathbf{p}_{i}^{*}, \mathbf{P}_{t h}\right)$ is short for an accuracy metric function that is dependent of the evaluation sample position, RSS model and deployment.

2) Solve $\mathbf{P}_{t h}^{\text {opt }}=$ optimize $\bar{f}\left(\mathbf{P}_{t h}\right)$, subject to certain constraints on $\mathbf{P}_{t h}$.

Lastly, devices that newly appear in the deployment area will be configured with the optimized reporting threshold, $\mathbf{P}_{t h}^{\text {opt }}$. The proximity reports can be used directly for positioning purposes, see for instance proximity report based particle filtering in [24], or serve as basis for analyzing user behaviors, such as dwell time and motion patterns. RSS threshold optimization is supported by an architecture, and we characterize the architecture by describing the logical entities performing different steps in the procedure. Furthermore, different node candidates are discussed for the logical entities.

Figure 3 shows the signaling chart of our proposed RSS thresholding procedure, where most of the key steps are performed by a computation entity. It can also be so that the offline processing is performed in a configuration entity, and the online processing in a separate fusion entity. Another possibility is that the calibration efforts are performed in a dedicated calibration entity. These logical entities can be implemented separately or jointly in a mobile device, a reference network node, or some other network node. The necessary communication in the two phases between a device and logical

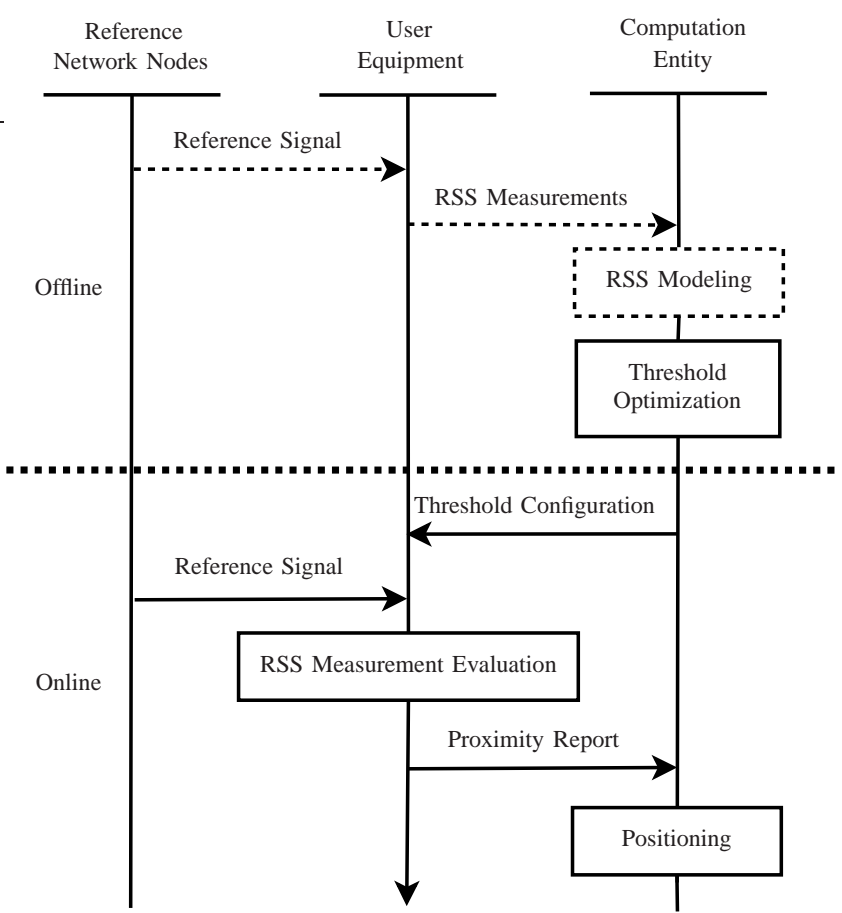

Fig. 3. Signaling chart of the proposed RSS thresholding procedure.

entity may be via a link with a reference network node, via some other communication link, or internally in the device.

Next, we provide more details about the implementation of the proposed RSS thresholding procedure, especially the optimization part.

- There exist many choices on the localization accuracy metric, for instance, the mean-square-error (MSE) and the localization outage probability of a specific proximity based position estimator, fundamental lower bounds on the MSE such as the Cramér-Rao bound (CRB) and the Barankin bound (BB). Bayesian type bounds [25] can also be used if the evaluation set contains a multiple of trajectories that follow certain motion model.

- We consider in the remainder of this paper the best achievable localization RMSE of unbiased, proximity report based position estimator, which is formulated in terms of the CRB or the BB derived in Section III. More precisely, we have

$$
f\left(\mathbf{p}_{i}^{*}, \mathbf{P}_{t h}\right) \triangleq \sqrt{\operatorname{tr}\left(\operatorname{CRB}\left(\mathbf{p}_{i}^{*}, \mathbf{P}_{t h}\right)\right)}
$$

or

$$
f\left(\mathbf{p}_{i}^{*}, \mathbf{P}_{t h}\right) \triangleq \sqrt{\operatorname{tr}\left(\mathrm{BB}\left(\mathbf{p}_{i}^{*}, \mathbf{P}_{t h}\right)\right)},
$$

where both the $\mathrm{CRB}$ and the $\mathrm{BB}$ on the estimation of $\mathbf{p}_{i}^{*}$ are computed in a snapshot manner for every sample position in the evaluation set.

- Practical constraints on the RSS thresholds may take into account the fact that the RSS thresholds, $P_{t h, i}$, $\forall i=1,2, \ldots, N$, are integer valued (due to quantization) and contained in an interval, say $\left[P_{t h, i}^{\min }, P_{t h, i}^{\max }\right]$, due to the sensor reading limits.

- Originally in [1], we set identical RSS thresholds $P_{t h, 1}=$ $P_{t h, 2}=\ldots=P_{t h, N}=P_{t h}$, test every possible RSS 
threshold candidate, and choose the one that minimizes the cost function. While this method does not apply to multiple RSS thresholds optimization due to the exponentially increased computational complexity as was explained in [1]. To sidestep this complex combinatorial problem, we first relax the integer constraint and resort to numerical optimization method to solve for an locally optimal set of RSS thresholds, $\mathbf{P}_{t h}^{\text {opt }}$, from the minimization problem. The result is then rounded to the nearest feasible integer.

- The cost function that minimizes the best achievable localization RMSE in the above example is not ensured to be convex in terms of the RSS thresholds. A numerical optimization method may stuck at local optimum.

- For the case that some reference nodes malfunction, principally, we need to redo RSS thresholding for the remaining nodes. While for the case that more reference nodes are to be added in a calibrated network, we need to consider where to place them in the first place. Intuitively, it is more meaningful to install them in the area where the localization accuracy is relatively poor. Besides, we need to collect RSS measurements and train the RSS model parameters for those newly installed reference nodes before we can redo RSS thresholds optimization. For large-scale sensor network, however, this is prohibitively expensive. In order to maintain similar performance with reasonable computation time, we can redo the optimization only for the 'neighbors' of the removed nodes in the former case or jointly for the newly added nodes and their 'neighbors' in the latter case.

\section{EXPERIMENTAL VALIDATION}

In Section IV, we have shown the whole procedure of optimizing RSS threshold(s) for enhanced overall localization performance. In what follows, we will validate our idea experimentally using real RSS measurements collected in an indoor bluetooth-low-energy (BLE) network. There is a big trend nowadays to use BLE networks for Internet of Things (IoT) due to the low power consumption and efficient monitoring capabilities of the BLE devices [26]. The optimized RSS thresholds not only lead to novel proximity based positioning methods but also enable user equipment (UE) assisted measurement to support handover, for instance, in Wideband Code Division Multiple Access (WCDMA) and Long-Term Evolution (LTE) systems [27], [28].

\section{A. Sensor Deployment, Measurement Campaign and Model Fitting}

Our experiments were conducted in a typical office environment at Ericsson Research, Linköping, Sweden. In total $N=12$ BLE beacons are placed uniformly in the area. The floor plan as well as the known beacon positions are illustrated using a local two-dimensional (2-D) coordinate system in Fig. 4. The BLE beacons serve as transmitters and broadcast data packages regularly. The transmit power is set to $P_{T}=-58 \mathrm{dBm}$ identically for all BLE beacons. Moderate scale measurement campaign was conducted during normal

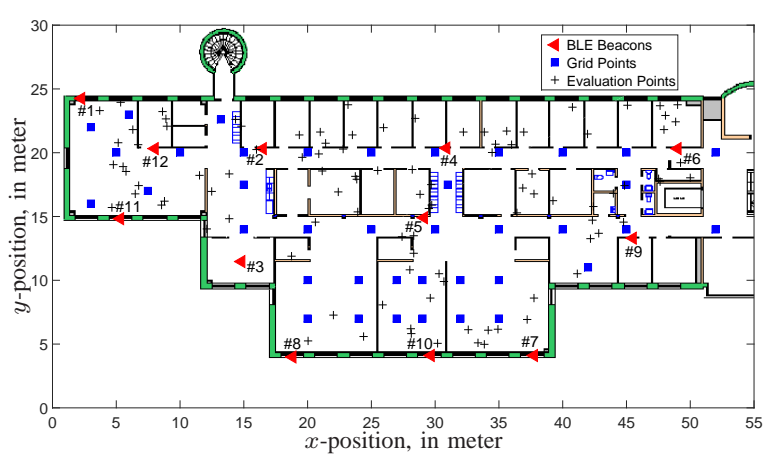

Fig. 4. Illustration of the floor plan where in total 12 BLE beacons are deployed, the preselected grids for online GPR modeling and the preselected evaluation set of test positions for RSS threshold optimization.

TABLE I

3-D POSITIONS (UNIT IN METER) OF 12 BLE BEACONS, THE AMOUNT OF RSS MEASUREMENTS (UNIT IN SAMPLES) COLLECTED BY EACH BLE BEACON AS THE OUTCOME OF THE MEASUREMENT CAMPAIGN AND THE CALIBRATED LINEAR LOG-DISTANCE MODEL PARAMETERS, $A, B, \sigma$ (UNIT IN $d \mathrm{~B})$.

\begin{tabular}{|l|c|c|c|}
\hline ID & Position $(x, y, z)$ & \# RSS Data & Parameters $(\hat{A}, \hat{B}, \hat{\sigma})$ \\
\hline$\# 1$ & $(2.27,24.26,2.60)$ & 1583 & $(-57.6,-2.5,6.9)$ \\
\hline$\# 2$ & $(16.47,20.34,2.35)$ & 2898 & $(-68.3,-1.8,7.2)$ \\
\hline$\# 3$ & $(14.83,11.48,0.71)$ & 2675 & $(-68.0,-1.9,6.5)$ \\
\hline$\# 4$ & $(30.89,20.36,2.35)$ & 3845 & $(-60.8,-2.3,7.5)$ \\
\hline$\# 5$ & $(29.13,14.90,2.54)$ & 4295 & $(-60.3,-2.4,6.9)$ \\
\hline$\# 6$ & $(49.00,20.34,2.35)$ & 1179 & $(-66.5,-1.9,6.7)$ \\
\hline$\# 7$ & $(37.74,4.10,2.60)$ & 2438 & $(-55.7,-2.6,6.5)$ \\
\hline$\# 8$ & $(18.82,4.00,2.60)$ & 642 & $(-73.0,-1.2,5.9)$ \\
\hline$\# 9$ & $(45.51,13.30,2.25)$ & 3014 & $(-61.9,-2.0,7.0)$ \\
\hline$\# 10$ & $(29.66,4.10,2.60)$ & 3024 & $(-57.6,-2.6,6.1)$ \\
\hline$\# 11$ & $(5.32,14.81,2.35)$ & 955 & $(-60.6,-2.6,7.2)$ \\
\hline$\# 12$ & $(8.00,20.32,2.60)$ & 1666 & $(-63.7,-1.9,6.5)$ \\
\hline
\end{tabular}

work hours. Throughout the measurement campaign, the UE walked along various predefined tracks and measured a total number of 28214 RSS measurements from the BLE beacons. For simpler Gaussian process regression model, the track positions are assumed to be precisely known. ${ }^{1}$ The obtained RSS measurements were eventually uploaded to a computation entity via Wi-Fi for RSS model fitting and threshold optimization. More details about the sensor deployment and the outcome of the measurement campaign are given in Table I.

We consider three RSS models, namely the linear logdistance model, the nonlinear full GPR model and the online GPR model, respectively. The linear model fitting follows the steps given in the first example of Section II and the calibrated model parameters are given in Table I. These parameters serve as our prior knowledge when choosing the initial guesses of the full GPR model parameters. The nonlinear full GPR model is learned with 600 RSS measurements randomly sampled from the full dataset. Herein, we only choose 600 measurements so that too long overall simulation time can be avoided for full GPR model calibration and RSS thresholds op-

\footnotetext{
${ }^{1}$ The track positions were essentially obtained from an app-based positioning algorithm developed by SenionLab and are supposed to be subject to zero mean Gaussian error with covariance matrix $\boldsymbol{\Sigma}_{\mathbf{p}}=\sigma_{p}^{2} \mathbf{I}_{2}$, where the standard deviation (std) is identical for both $x$ - and $y$-direction and varies moderately from $10^{-4}$ to 2.6 meter for different positions.
} 


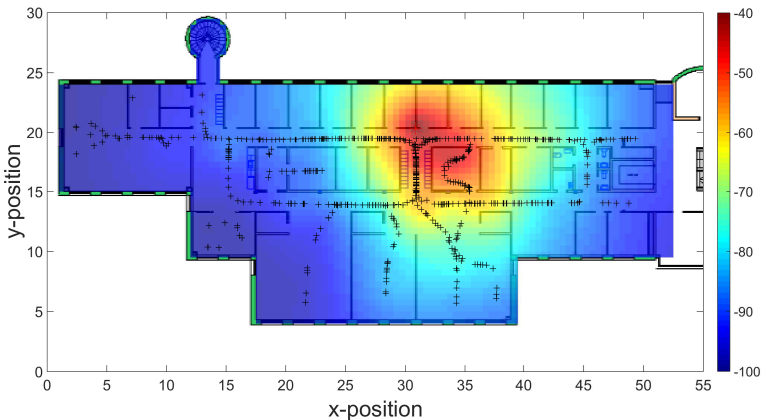

(a)

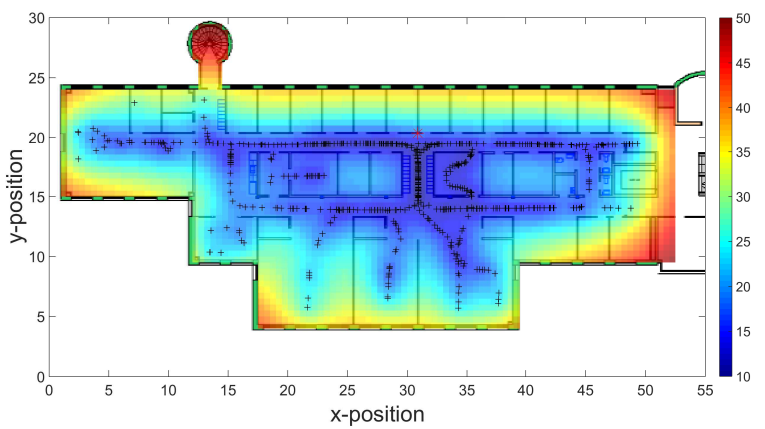

(b)

Fig. 5. Illustration of the training data set (600 samples marked by black + ) and the calibrated full GPR model for the 4th BLE beacon: (a) depicts the posterior mean of (9) and (b) depicts the posterior variance of (9).

timization. We show both the posterior mean and the posterior covariance for the fourth BLE beacon in Fig. 5. In contrast, due to the significantly reduced computational complexity, we use the full dataset to train the online GPR model and use it in the RSS threshold optimization. Herein, a number of 39 grids are predefined in the online GPR model and they are depicted in Fig. 4. In Fig. 6, we show similar results for the online GPR model. When comparing Fig. 5 with Fig. 6, it is observed that posterior mean map of the online GPR model is richer in context than that of the full GPR model. This might be due to larger number of measurements used in the modeling. However, the posterior covariance is accurate only at the predefined grids and needs to be interpolated at other points. Hence, if the evaluation set contains a lot of sample positions that are far from the grid points, we will probably suffer from higher approximation error.

Lastly, we note that due to space limitation, we are unable to show the posterior mean and covariance for other beacons. The results for other BLE beacons are similar. It is clear from Fig. 5 that the energy pattern is not isotropic. To the left side of the fourth BLE beacon is concrete wall and the energy is highly absorbed. While to its right side, the energy is much less absorbed owing to the wooden wall. These explain why we did not see a uniform, isotropic energy pattern. This is the advantage of the GP model over empirical models as the data speak for the model.

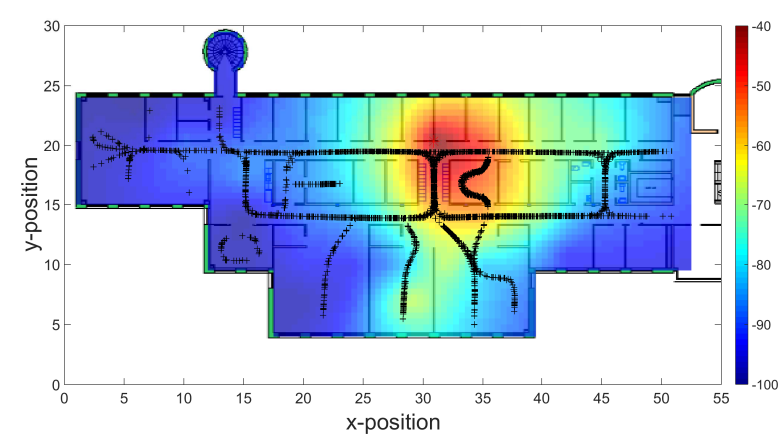

(a)

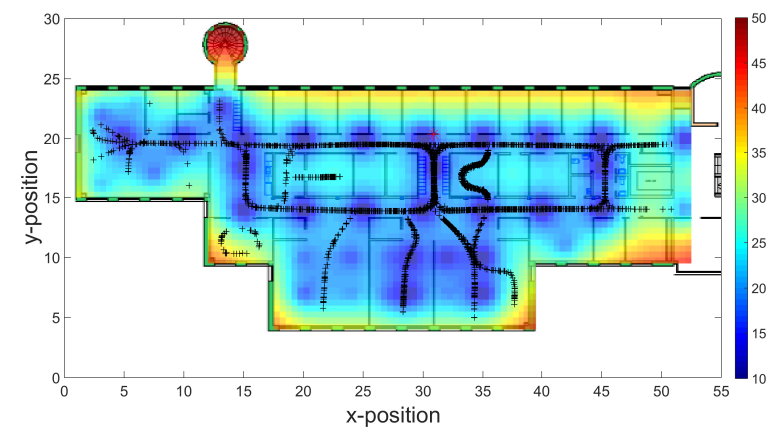

(b)

Fig. 6. Illustration of the training data set (3845 samples marked by black + ) and the calibrated online GPR model for the 4th BLE beacon: (a) depicts the posterior mean of (13) and (b) depicts the posterior variance of (13).

\section{B. RSS Threshold Optimization}

Before performing RSS threshold optimization, we first generate a set $\mathcal{X}^{*}$ of 100 evaluation positions, $\mathbf{p}_{i}^{*}$, uniformly in the deployment area, as shown in Fig. $4 .^{2}$ The weighting factors are set equally as $w_{i}^{*}=1 /\left|\mathcal{X}^{*}\right|$ for all evaluation positions in $\mathcal{X}^{*}$.

As the localization accuracy metric, we compute the best achievable RMSE either in terms of the CRB or the BB. Herein, we assume that the $z$-component of all sample positions is fixed to 1.3 meter and known a priori. Thus, we only concern about position estimation in $x$ - and $y$-directions. As a consequence, $f\left(\mathbf{p}_{i}^{*}, \mathbf{P}_{t h}\right)$ boils down to $f\left(\left[x_{i}^{*}, y_{i}^{*}\right], \mathbf{P}_{t h}\right)$. Our focus is to optimize a RSS threshold individually for each BLE beacon and compare the overall localization performance with that led by a global RSS threshold. We use the MATLAB function fmincon to solve the multi-variate minimization problem with the cost function given in (34) and the constraints $-100 \mathrm{dBm} \leq P_{t h, i} \leq-70 \mathrm{dBm}, i=1,2, \ldots, 12$. Afterwards, we round the optimized RSS thresholds, $\mathbf{P}_{t h}^{\text {opt }}$ to the nearest integers. The starting point is selected to be $P_{t h, 1}=P_{t h, 2}=$ $\ldots=P_{t h, 12}=-90 \mathrm{dBm}$. The number of test points, $M_{b}$, for computing the $\mathrm{BB}$ is set to 25 to save computational time.

We depict the multiple RSS thresholds optimized individually for each BLE beacon versus the global RSS threshold

\footnotetext{
${ }^{2}$ In contrast to [1], we used here a smaller number of evaluation positions that well represent the deployment area. The final results do not differ much, but the computation cost can be reduced significantly, especially for the BB based RSS thresholds optimization.
} 


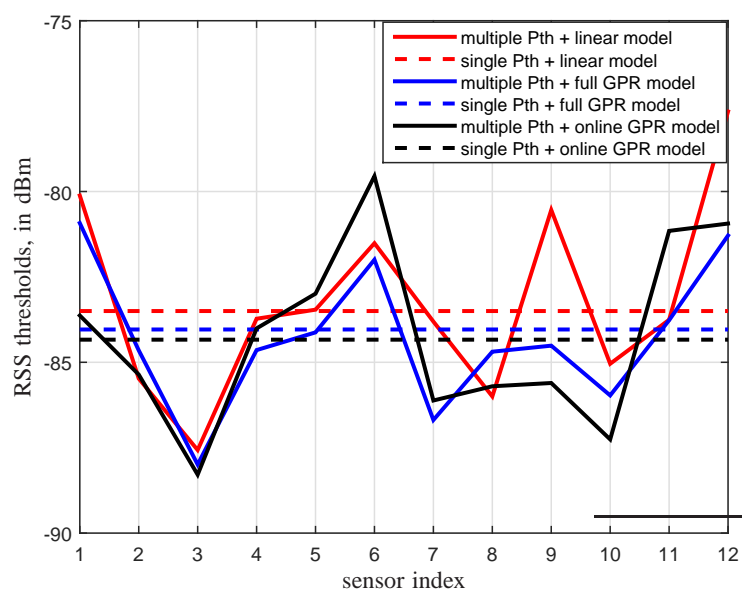

(a)

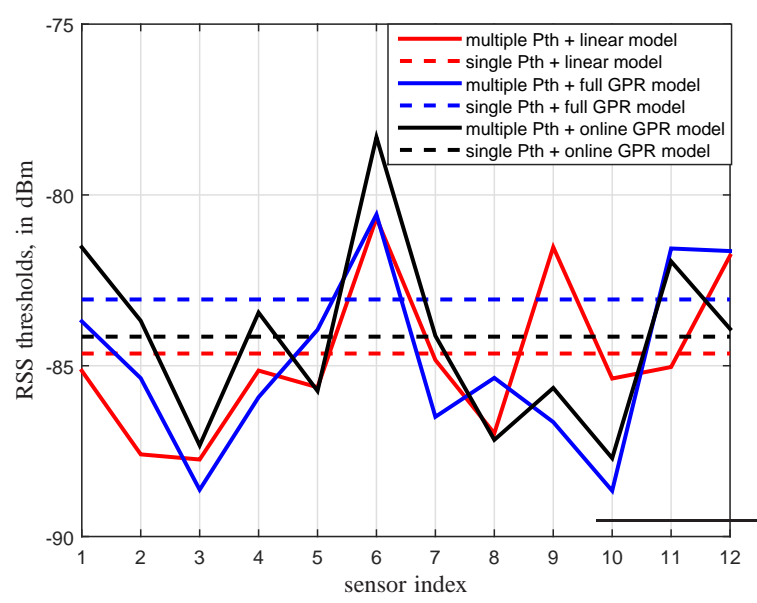

(b)

Fig. 7. Illustration of multiple RSS thresholds optimized individually for each BLE beacon versus the global RSS threshold optimized for all BLE beacons: (a) CRB based (b) BB based.

optimized for all beacons based on the CRB in Fig. 7(a) and based on the BB in Fig. 7(b). To give better a vision, we did not use the rounded values in these figures. Interestingly, the average of the multiple RSS thresholds $\bar{P}_{t h}^{\text {opt }} \triangleq \frac{1}{N} \sum_{i=1}^{N} P_{t h, i}^{\text {opt }}$ is very close to the global RSS threshold in both cases. The global RSS threshold does not differ much for different combinations of RSS model and lower bound. The improvement becomes more obvious when we decrease the number of BLE beacons in the network. For instance, when we merely use Beacon 5, 6, 12, the difference will be around one meter. In these figures, it is also obvious that advanced GPR based RSS models help improve the positioning accuracy about two meters. The positioning performance is comparable for the two GPR models, but the computational time of the online GPR model is about 50 times faster than that of the full GPR model. Furthermore, we show the overall best achievable RMSE for different combinations of RSS model and lower bound in Fig. 8. Only modest performance improvement has been obtained by using the RSS threshold optimized individually for each beacon. However, much more computational time has

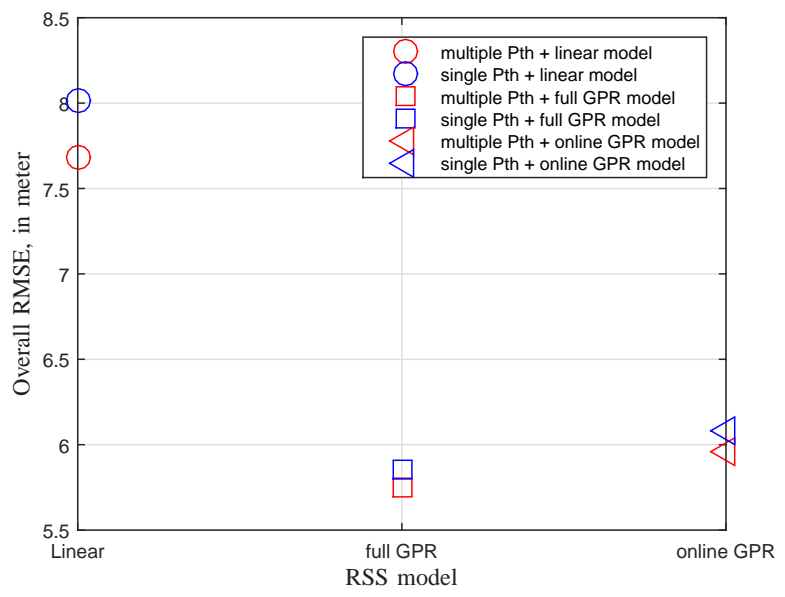

(a)

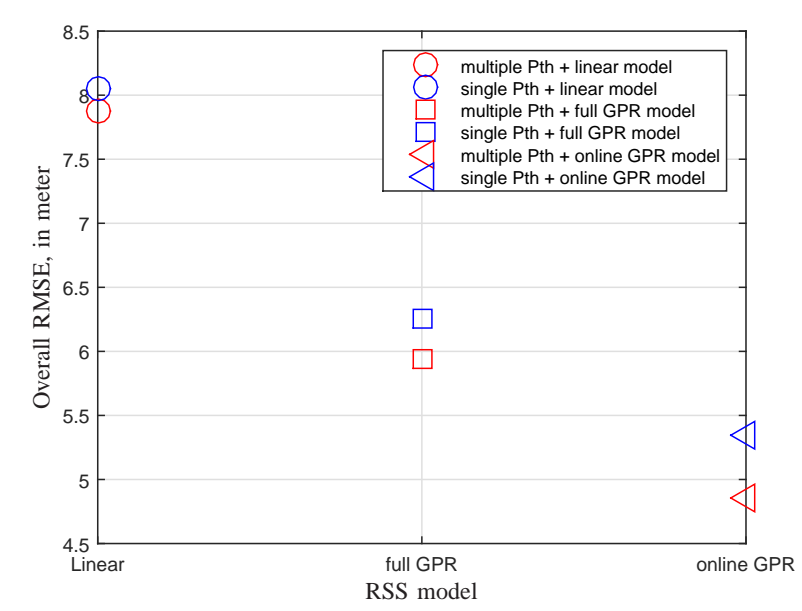

(b)

Fig. 8. Illustration of the overall best achievable RMSE using multiple RSS thresholds optimized individually for each BLE beacon versus the global RSS threshold optimized for all BLE beacons (a) CRB based (b) BB based.

also been observed when optimizing multiple RSS thresholds. The RMSE led by the BB based RSS thresholds is higher than the CRB based counterparts. Similar to the conclusion drawn in [2], the BB is less optimistic about the mean-square-error of proximity based position estimator than the CRB.

Next, we aim to show the improvement made when using an optimal RSS threshold as compared to a suboptimal one. Herein, we simply consider the global RSS threshold for three different RSS models. In Fig. 9, we depict the overall best achievable localization RMSE as a function of the RSS threshold $P_{t h}$, which ranges from $-100 \mathrm{dBm}$ to $-70 \mathrm{dBm}$ with an increment $1 \mathrm{dBm}$. As is shown in the figure, the minimum is achieved at $-83 \mathrm{dBm},-84 \mathrm{dBm}$, and $-84 \mathrm{dBm}$ respectively for the three different RSS models. The optimal thresholds coincide with those given in Fig. 7(a) if they are rounded to the nearest integer therein. The corresponding RMSE values coincide with those shown in Fig. 8(a) for the CRB case. It is not surprising to see the convex profile of the RMSE curves in all cases. The reason is that too large or too small threshold gives very little information about an unknown location. We 


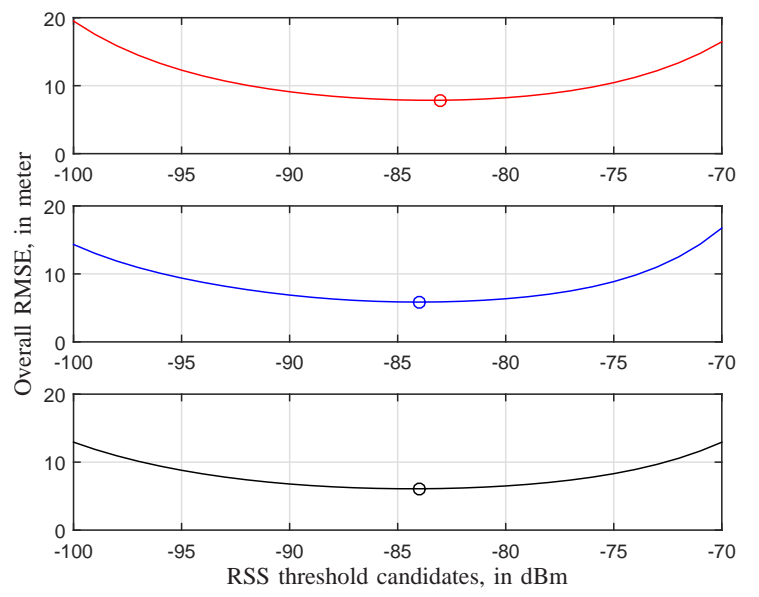

Fig. 9. Overall best achievable localization RMSE (in terms of CRB) versus threshold candidates for the linear log-distance model in subfigure-I, nonlinear full GPR model in subfigure-II, and nonlinear online GPR model in subfigure III, respectively.

have explained this with a toy example in the introduction. A formal proof of the convexity of our cost function might be interesting for our future work.

Lastly, we demonstrate a target tracking example using the trained RSS thresholds. More precisely, particle filtering has been applied to obtain the position estimates for a specific trajectory as shown in Figure 10. The trajectory is selected from one of the 52 predefined tracks. It is noted that with the full GPR-based RSS measurement model, the best positioning performance has been achieved. While with the full GPRbased proximity measurement model, less accurate position estimates have been obtained. Nevertheless, the two corridors on this floor can be successfully distinguished in all cases. This is important for determining pedestrian flow required in various indoor applications. To compare the statistical performance of the different measurement models and different thresholding algorithms, the cumulative distribution function (CDF) of the estimation errors are compared in Figure 11. It is clear that the median positioning error for RSS model is around 1.3 meter. For proximity model with global threshold, the median error is approximate 2.3 meter and for proximity model with optimized thresholds from $\mathrm{CRB}$ and $\mathrm{BB}$, there is around 0.3 meter improvement. With the optimized thresholds from $\mathrm{BB}$, the position accuracy has been improved for most of the cases, while for around $20 \%$ of the cases large position errors have been observed.

\section{CONCLUSiOnS}

We have introduced a generic framework of RSS threshold optimization for indoor sensor networks. With the aid of this framework, we can obtain the most informative proximity report by means of optimizing the overall localization accuracy in a deployment area. Two pivotal building elements of this work are the RSS model and the fundamental lower bound. We have tested both the full GPR based model and an online GPR based model. The computational complexity of training these two models scales as $\mathcal{O}\left(M^{3}\right)$ and $\mathcal{O}\left(s^{2} M\right)$,

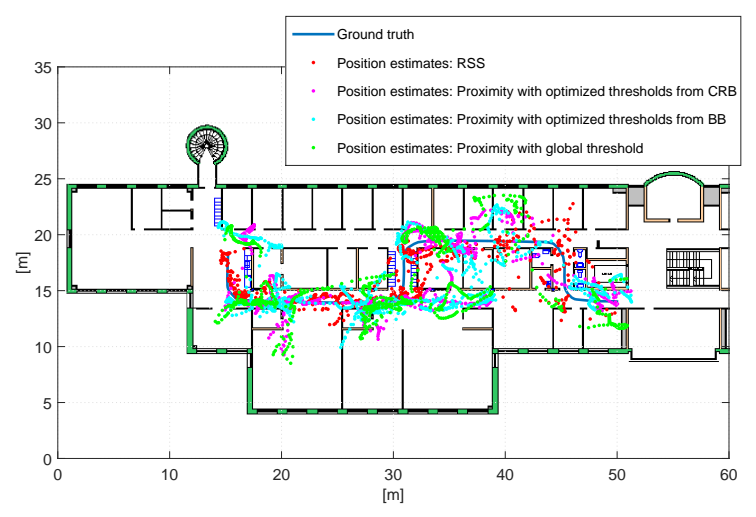

Fig. 10. Illustration of the estimated positions of an exemplary track.

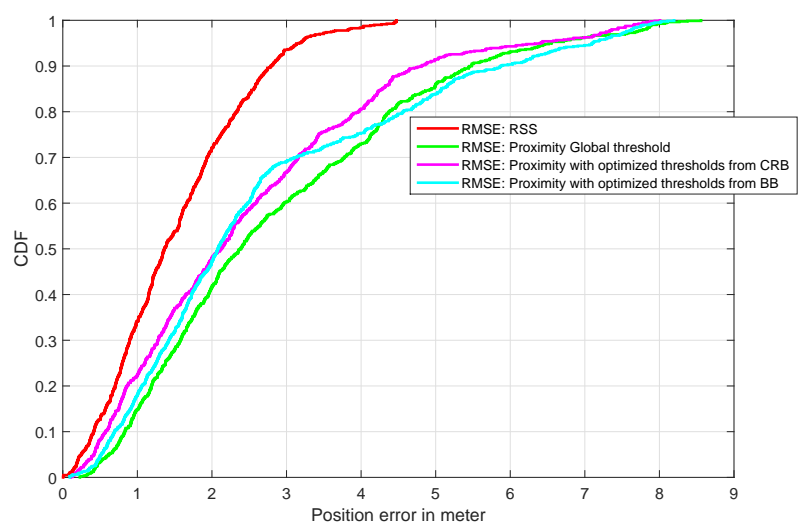

Fig. 11. CDF of the positioning error of an exemplary track.

respectively. As our localization accuracy metric, the best achievable positioning RMSE is expressed either in terms of the Cramér-Rao Bound or the Barankin Bound. The computational complexity of the CRB scales $\mathcal{O}\left(N M^{2}\right)$ for the full GPR model and $\mathcal{O}\left(s^{2} N\right)$ for the online GPR model. The computational complexity of the BB scales as $\mathcal{O}(N K)+\mathcal{O}\left(M_{b}^{3}\right)$. Large number of test points for computing the $\mathrm{BB}$ is hence computationally prohibitive. We tested different combinations of RSS model and lower bound in various experiments with real data collected from a live BLE network deployed at Ericsson Research. We compared the performance of using different thresholds for different reference nodes and using a single threshold for all reference nodes. The results confirmed that the former modality outperforms the latter, but for large scale sensor network the improvement can be negligible.

\section{APPENDIX A}

The maximum-likelihood estimate of the GPR model parameters, $\hat{\boldsymbol{\theta}}_{i}$, can be obtained by maximizing the Gaussian prior likelihood function, cf.(7), with respect to $\boldsymbol{\theta}_{i}$, which is equivalent to

$$
\arg \min _{\boldsymbol{\theta}_{i}} g\left(\boldsymbol{\theta}_{i}\right) \triangleq\left(\mathbf{r}_{i}-\mathbf{m}_{i}\right)^{T} \mathbf{C}_{i}^{-1}\left(\mathbf{r}_{i}-\mathbf{m}_{i}\right)+\ln \left|\mathbf{C}_{i}\right| .
$$

For clarity, we note that the optimization variables are in the mean vector $\mathbf{m}_{i}$ and covariance matrix $\mathbf{C}_{i}$. More precisely, 
$A_{i}$ and $B_{i}$ are in the mean vector $\mathbf{m}_{i}$, while $\sigma_{s, i}^{2}, \sigma_{n, i}^{2}$ and $l_{c, i}$ are in the covariance matrix $\mathbf{C}_{i}$.

Various existing numerical methods can be adopted to solve this minimization problem, such as the limited-memory BFGS (LBFGS) quasi-Newton method [11] and the conjugate gradient (CG) method. Herein, we adopt the former method which requires the first-order derivatives of the cost function, $g\left(\boldsymbol{\theta}_{i}\right)$. Equation (37) is essentially convex with respect to the parameters. Similar proof can be found in [29, Section 4.8.2]. Due to space limitation, we only give the results as follows:

$$
\begin{aligned}
& \frac{\partial g\left(\boldsymbol{\theta}_{i}\right)}{\partial A_{i}}=\mathbf{a}_{i}\left(\mathbf{C}_{i}^{-1}+\left(\mathbf{C}_{i}^{-1}\right)^{T}\right)\left(\mathbf{r}_{i}-\mathbf{m}_{i}\right) \\
& \frac{\partial g\left(\boldsymbol{\theta}_{i}\right)}{\partial B_{i}}=\mathbf{b}_{i}\left(\mathbf{C}_{i}^{-1}+\left(\mathbf{C}_{i}^{-1}\right)^{T}\right)\left(\mathbf{r}_{i}-\mathbf{m}_{i}\right) \\
& \frac{\partial g\left(\boldsymbol{\theta}_{i}\right)}{\partial \sigma_{s, i}^{2}}=\operatorname{tr}\left\{\left[\mathbf{C}_{i}^{-1}-\left(\mathbf{C}_{i}^{-1}\left(\mathbf{r}_{i}-\mathbf{m}_{i}\right)\right)(\cdot)^{T}\right] \frac{\partial \mathbf{C}_{i}}{\partial \sigma_{s, i}^{2}}\right\} \\
& \frac{\partial g\left(\boldsymbol{\theta}_{i}\right)}{\partial l_{c, i}}=\operatorname{tr}\left\{\left[\mathbf{C}_{i}^{-1}-\left(\mathbf{C}_{i}^{-1}\left(\mathbf{r}_{i}-\mathbf{m}_{i}\right)\right)(\cdot)^{T}\right] \frac{\partial \mathbf{C}_{i}}{\partial l_{c, i}}\right\} \\
& \frac{\partial g\left(\boldsymbol{\theta}_{i}\right)}{\partial \sigma_{n, i}^{2}}=\operatorname{tr}\left\{\left[\mathbf{C}_{i}^{-1}-\left(\mathbf{C}_{i}^{-1}\left(\mathbf{r}_{i}-\mathbf{m}_{i}\right)\right)(\cdot)^{T}\right] \frac{\partial \mathbf{C}_{i}}{\partial \sigma_{n, i}^{2}}\right\},
\end{aligned}
$$

where

$$
\begin{aligned}
\mathbf{a}_{i} & \triangleq \frac{\partial\left(\mathbf{r}_{i}-\mathbf{m}_{i}\right)^{T}}{\partial A_{i}}=-\mathbf{1}^{T}, \\
\mathbf{b}_{i} & \triangleq \frac{\partial\left(\mathbf{r}_{i}-\mathbf{m}_{i}\right)^{T}}{\partial B_{i}}=-10 \cdot\left[\log d_{i, 1}, \ldots, \log d_{i, M}\right] \\
{\left[\frac{\partial \mathbf{C}_{i}}{\partial \sigma_{s, i}^{2}}\right]_{j, k} } & = \begin{cases}1, & j=k \\
\exp \left[\frac{-\left\|\mathbf{p}_{i, j}-\mathbf{p}_{i, k}\right\|}{l_{c, i}}\right], & j \neq k\end{cases} \\
{\left[\frac{\partial \mathbf{C}_{i}}{\partial l_{c, i}}\right]_{j, k} } & = \begin{cases}0, & j=k \\
\sigma_{s, i}^{2} \exp \left[\frac{-\left\|\mathbf{p}_{i, j}-\mathbf{p}_{i, k}\right\|}{l_{c, i}}\right] \frac{\left\|\mathbf{p}_{i, j}-\mathbf{p}_{i, k}\right\|}{l_{c, i}^{2}}, & j \neq k\end{cases} \\
\frac{\partial \mathbf{C}_{i}}{\partial \sigma_{n, i}^{2}} & =\mathbf{I}_{M} .
\end{aligned}
$$

Here we use $(A)(\cdot)^{T}$ to denote $(A)(A)^{T}$ for brevity.

\section{APPENDIX B}

Imagine that $\mathcal{D}_{g, i}=\left\{\overline{\mathbf{P}}, \overline{\mathbf{r}}_{i}\right\}$ is also a training data set despite that $\overline{\mathbf{r}}_{i}$ is latent. Given a novel input, $\mathbf{p}_{*}$, the posterior distribution of observing a noisy RSS $r_{i}\left(\mathbf{p}_{*}\right)$, given $\mathcal{D}_{g, i}$, can be easily obtained as follows:

$$
p\left(r_{i}\left(\mathbf{p}_{*}\right) \mid \mathbf{p}_{*}, \mathcal{D}_{g, i}\right) \sim \mathcal{N}\left(\mu_{i, g}^{p},\left(\sigma_{i, g}^{p}\right)^{2}\right),
$$

where

$$
\begin{aligned}
\mu_{i, g}^{p} & =\mathbf{k}_{i}\left(\mathbf{p}_{*}, \overline{\mathbf{P}}\right)^{T} \overline{\mathbf{K}}_{i}^{-1}\left(\overline{\mathbf{r}}_{i}-\overline{\mathbf{m}}_{i}\right)+m_{i}\left(\mathbf{p}_{*}\right) \\
\sigma_{i, g}^{2, p} & =\sigma_{s, i}^{2}+\sigma_{n, i}^{2}-\mathbf{k}_{i}\left(\mathbf{p}_{*}, \overline{\mathbf{P}}\right)^{T} \overline{\mathbf{K}}_{i}^{-1} \mathbf{k}_{i}\left(\mathbf{p}_{*}, \overline{\mathbf{P}}\right) .
\end{aligned}
$$

The posterior distribution of $r_{i}\left(\mathbf{p}_{*}\right)$, given $\mathcal{D}_{i}$ and $\overline{\mathbf{P}}$, can be computed analytically via the following marginalization:

$p\left(r_{i}\left(\mathbf{p}_{*}\right) \mid \mathbf{p}_{*}, \mathcal{D}_{i}, \overline{\mathbf{P}}\right)=\int p\left(\overline{\mathbf{r}}_{i} \mid \overline{\mathbf{P}}, \mathcal{D}_{i}\right) p\left(r_{i}\left(\mathbf{p}_{*}\right) \mid \mathbf{p}_{*}, \mathcal{D}_{g, i}, \mathcal{D}_{i}\right) \mathrm{d} \overline{\mathbf{r}}_{i}$,

and approximated with reduced computational complexity, like in [30], by

$$
p\left(r_{i}\left(\mathbf{p}_{*}\right) \mid \mathbf{p}_{*}, \mathcal{D}_{i}, \overline{\mathbf{P}}\right) \approx \int p\left(\overline{\mathbf{r}}_{i} \mid \overline{\mathbf{P}}, \mathcal{D}_{i}\right) p\left(r_{i}\left(\mathbf{p}_{*}\right) \mid \mathbf{p}_{*}, \mathcal{D}_{g}\right) \mathrm{d} \overline{\mathbf{r}}_{\mathrm{i}} .
$$

Since both $p\left(r_{i}\left(\mathbf{p}_{*}\right) \mid \mathbf{p}_{*}, \mathcal{D}_{g, i}\right)$ and $p\left(\overline{\mathbf{r}}_{i} \mid \overline{\mathbf{P}}, \mathcal{D}_{i}\right)$ are Gaussian distributed, applying Lemma A.1 in [31] yields eventually (14a) and (14b).

\section{APPENDIX C}

Log-distance Model: The first-order derivatives of $G_{i}(\mathbf{p})$ can be derived for any $m \in\{x, y, z\}$ as follows:

$$
\begin{aligned}
\frac{\partial G_{i}(\mathbf{p})}{\partial m} & \equiv \frac{\partial}{\partial m}\left\{\frac{1}{2} \operatorname{erf}\left(\frac{P_{t h, i}-\hat{\mu}_{i}(\mathbf{p})}{\sqrt{2} \hat{\sigma}_{i}}\right)\right\} \\
& =\frac{-10 \hat{B}_{i}}{\sqrt{2 \pi} \hat{\sigma}_{i} \ln 10} \exp \left[\frac{\left(P_{t h, i}-\hat{\mu}_{i}(\mathbf{p})\right)^{2}}{-2 \hat{\sigma}_{i}^{2}}\right] \frac{m-m_{\mathrm{r}, i}}{\left\|\mathbf{p}-\mathbf{p}_{\mathrm{r}, i}\right\|^{2}} .
\end{aligned}
$$

Inserting the above result into (26) and performing some algebraic manipulations yields

$$
f_{i, m n}=\gamma_{i} \cdot \frac{\left(m-m_{\mathrm{r}, i}\right)\left(n-n_{\mathrm{r}, i}\right)}{\left\|\mathbf{p}-\mathbf{p}_{\mathrm{r}, i}\right\|^{4}}
$$

where $\gamma_{i}$ is a variable in terms of the calibrated RSS model parameters and the RSS threshold, more precisely,

$$
\gamma_{i}=\frac{200 \hat{B}_{i}^{2}}{\pi \hat{\sigma}_{i}^{2} \ln ^{2}(10)} \cdot \frac{\exp \left[\frac{-\left(P_{t h, i}-\hat{\mu}_{i}(\mathbf{p})\right)^{2}}{\hat{\sigma}_{i}^{2}}\right]}{1-\operatorname{erf}^{2}\left(\frac{P_{t h, i}-\hat{\mu}_{i}(\mathbf{p})}{\sqrt{2} \hat{\sigma}_{i}}\right)} .
$$

Full GPR model: The first-order derivatives can be derived for the full GPR model with a bit more efforts as follows:

$$
\begin{aligned}
\frac{\partial G_{i}(\mathbf{p})}{\partial m} & \equiv \frac{\partial}{\partial m}\left\{\frac{1}{2} \operatorname{erf}\left(\frac{P_{t h, i}-\hat{\mu}_{i}(\mathbf{p})}{\sqrt{2} \hat{\sigma}_{i}(\mathbf{p})}\right)\right\} \\
& =\gamma_{i} \cdot\left[\frac{\frac{\partial u_{i}(\mathbf{p})}{\partial m} v_{i}(\mathbf{p})-\frac{\partial v_{i}(\mathbf{p})}{\partial m} u_{i}(\mathbf{p})}{v_{i}^{2}(\mathbf{p})}\right],
\end{aligned}
$$

where

$$
\begin{aligned}
\gamma_{i} & =\frac{1}{\sqrt{\pi}} \exp \left[-\frac{\left(P_{t h, i}-\hat{\mu}_{i}(\mathbf{p})\right)^{2}}{2 \hat{\sigma}_{i}^{2}(\mathbf{p})}\right], \\
u_{i}(\mathbf{p}) & =P_{t h}-\hat{\mu}_{i}(\mathbf{p}), \\
v_{i}(\mathbf{p}) & =\sqrt{2} \hat{\sigma}_{i}(\mathbf{p}), \\
\frac{\partial u_{i}(\mathbf{p})}{\partial m} & =-\frac{\partial \hat{\mu}_{i}(\mathbf{p})}{\partial m}, \\
\frac{\partial v_{i}(\mathbf{p})}{\partial m} & =\frac{1}{\sqrt{2 \hat{\sigma}_{i}^{2}(\mathbf{p})}} \frac{\partial \hat{\sigma}_{i}^{2}(\mathbf{p})}{\partial m} .
\end{aligned}
$$


In this case $\hat{\mu}_{i}(\mathbf{p})$ and $\hat{\sigma}_{i}^{2}(\mathbf{p})$ are given in (10a) and (10b). Furthermore, their derivatives are calculated as follows:

$$
\begin{gathered}
\frac{\partial \hat{\mu}_{i}(\mathbf{p})}{\partial m}=\frac{\partial \mathbf{k}_{i}\left(\mathbf{p}, \mathbf{P}_{i}\right)}{\partial m} \mathbf{C}_{i}^{-1}\left(\mathbf{y}_{i}-\mathbf{m}_{i}\right)+\frac{10 B_{i}}{\ln 10} \frac{m-m_{r, i}}{\left\|\mathbf{p}-\mathbf{p}_{r, i}\right\|^{2}}, \\
\frac{\partial \hat{\sigma}_{i}^{2}(\mathbf{p})}{\partial m}=-\frac{\partial \mathbf{k}_{i}\left(\mathbf{p}, \mathbf{P}_{i}\right)}{\partial m}\left(\mathbf{C}_{i}^{-1}+\left(\mathbf{C}_{i}^{-1}\right)^{T}\right) \mathbf{k}_{i}\left(\mathbf{p}, \mathbf{P}_{i}\right),
\end{gathered}
$$

where $\frac{\partial \mathbf{k}_{i}\left(\mathbf{p}, \mathbf{P}_{i}\right)}{\partial m}$ is a vector of size $M \times 1$ with the $j$ th entry given by

$$
\left[\frac{\partial \mathbf{k}_{i}\left(\mathbf{p}, \mathbf{P}_{i}\right)}{\partial m}\right]_{j}=\frac{-\sigma_{s, i}^{2}}{l_{c, i}} \exp \left[\frac{-\left\|\mathbf{p}-\mathbf{p}_{i, j}\right\|}{l_{c, i}}\right] \frac{\left(m-m_{i, j}\right)}{\left\|\mathbf{p}-\mathbf{p}_{i, j}\right\|} .
$$

Having the results (10a), (10b), (49a) and (49b), we can then easily evaluate $u_{i}(\mathbf{p}), v_{i}(\mathbf{p}), \frac{\partial u_{i}(\mathbf{p})}{\partial m}, \frac{\partial v_{i}(\mathbf{p})}{\partial m}$ via (48), and eventually the derivative of $G_{i}(\mathbf{p})$ via (47).

Online GPR model: The derivations are similar to those shown above for the full GPR model. In this case $\hat{\mu}_{i}(\mathbf{p})$ and $\hat{\sigma}_{i}^{2}(\mathbf{p})$ are given in (14a) and (14b). Similarly, their derivatives are calculated as follows:

$$
\begin{gathered}
\frac{\partial \hat{\mu}_{i}(\mathbf{p})}{\partial m}=\frac{\partial \mathbf{k}_{i}(\mathbf{p}, \overline{\mathbf{P}})}{\partial m} \overline{\mathbf{K}}_{i}^{-1}\left(\boldsymbol{\mu}_{i, M}^{g}-\overline{\mathbf{m}}_{i}\right)+\frac{10 B_{i}}{\ln 10} \frac{m-m_{r, i}}{\| \mathbf{p}-\underset{(51 \mathrm{a})}{\mathbf{p}_{r, i} \|^{2}}} \\
\frac{\partial \hat{\sigma}_{i}^{2}(\mathbf{p})}{\partial m}=-\frac{\partial \mathbf{k}_{i}(\mathbf{p}, \overline{\mathbf{P}})}{\partial m}\left(\overline{\mathbf{K}}_{i}^{-1}+\left(\overline{\mathbf{K}}_{i}^{-1}\right)^{T}\right) \mathbf{k}_{i}(\mathbf{p}, \overline{\mathbf{P}}), \quad(51 \mathrm{~b})
\end{gathered}
$$

where $\frac{\partial \mathbf{k}_{i}(\mathbf{p}, \overline{\mathbf{P}})}{\partial m}$ is a vector of size $s \times 1$ with the $j$ th entry given by

$$
\left[\frac{\partial \mathbf{k}_{i}(\mathbf{p}, \overline{\mathbf{P}})}{\partial m}\right]_{j}=\frac{-\sigma_{s, i}^{2}}{l_{c, i}} \exp \left[\frac{-\left\|\mathbf{p}-\overline{\mathbf{p}}_{j}\right\|}{l_{c, i}}\right] \frac{\left(m-m_{j}\right)}{\left\|\mathbf{p}-\overline{\mathbf{p}}_{j}\right\|} .
$$

Having the results (14a), (14b), (51a) and (51b), we can then easily evaluate $u_{i}(\mathbf{p}), v_{i}(\mathbf{p}), \frac{\partial u_{i}(\mathbf{p})}{\partial m}, \frac{\partial v_{i}(\mathbf{p})}{\partial m}$ via (48), and eventually the derivative of $G_{i}(\mathbf{p})$ via (47).

\section{REFERENCES}

[1] F. Yin, Y. Zhao, and F. Gunnarsson, "Proximity report triggering threshold optimization for network-based indoor positioning," in Proc. Int. Conf. on Information Fusion, Washington D.C., USA, July 2015, pp. 1061-1069.

[2] — - "Fundamental bounds on position estimation using proximity reports," in submitted to IEEE Int. Conf. Vehicular Technology (VTC), 2016.

[3] F. Gustafsson, Statistical Sensor Fusion, 2nd ed. Lund, Sweden: Studentlitteratur, 2012.

[4] N. Bulusu, J. Heidemann, and D. Estrin, "GPS-less low-cost outdoor localization for very small devices," IEEE Personal Communications, vol. 7 , no. 5, pp. 28-34, Oct. 2000.

[5] T. He, C. Huang, B. M. Blum, J. A. Stankovic, and T. Abdelzaher, "Range-free localization schemes for large scale sensor networks," in Proc. Annual International Conference on Mobile Computing and Networking. New York, NY, USA: ACM, 2003, pp. 81-95.

[6] N. Patwari and A. O. Hero, III, "Using proximity and quantized rss for sensor localization in wireless networks," in Proc. ACM Int. Conf. on Wireless Sensor Networks and Applications. New York, NY, USA: ACM, 2003, pp. 20-29.

[7] K. Yedavalli, B. Krishnamachari, S. Ravula, and B. Srinivasan, "Ecolocation: a sequence based technique for RF localization in wireless sensor networks," in Proc. Information Processing in Sensor Networks (IPSN), Los Angeles, California, USA, April 2005, pp. 285-292.
[8] N. A. M. Maung and M. Kawai, "Performance improvement of connectivity-based localization using iterative learning," in Proc. Int. Conf. Localization and GNSS (ICL-GNSS), Helsinki, Finland, June 2014, pp. 1-6.

[9] G. Giorgetti, S. K. S. Gupta, and G. Manes, "Optimal RSS threshold selection in connectivity-based localization schemes," in Proc. Int. Symp. on Modeling, Analysis and Simulation of Wireless and Mobile Systems. New York, NY, USA: ACM, 2008, pp. 220-228.

[10] A. Goldsmith, Wireless Communications. New York, NY, USA: Cambridge University Press, 2006.

[11] C. E. Rasmussen and C. K. I. Williams, Gaussian Processes for Machine Learning. Cambridge, MA, USA: MIT Press, 2006.

[12] M. Gudmundson, "Correlation model for shadow fading in mobile radio systems," Electronics Letters, vol. 27, no. 23, pp. 2145-2146, Nov. 1991.

[13] A. Schwaighofer, M. Grigoras, V. Tresp, and C. Hoffmann, GPPS: A Gaussian process positioning system for cellular networks, ser. Advances in Neural Information Processing Systems. MIT Press, 2004, no. 16.

[14] B. Ferris, D. Hhnel, and D. Fox, "Gaussian processes for signal strengthbased location estimation," in Proc. Robotics Science and Systems, 2006.

[15] R. Di Taranto, S. Muppirisetty, R. Raulefs, D. Slock, T. Svensson, and H. Wymeersch, "Location-aware communications for 5G networks: How location information can improve scalability, latency, and robustness of 5G," IEEE Signal Process. Mag., vol. 31, no. 6, pp. 102-112, Nov. 2014.

[16] J. Quiñonero Candela and C. E. Rasmussen, "A unifying view of sparse approximate Gaussian process regression," J. Mach. Learn. Res., vol. 6, pp. 1939-1959, Dec. 2005.

[17] M. F. Huber, "Recursive Gaussian process regression," in Proc. Int. Conf. on Acoustics, Speech and Signal Processing (ICASSP), May 2013, pp. $3362-3366$.

[18] M. Huber, "Recursive Gaussian process: On-line regression and learning," Pattern Recognition Letters, vol. 45, pp. 85-91, 2014.

[19] J. S. Abel, "A bound on mean-square-estimate error," IEEE Trans. Information Theory, vol. 39, no. 5, pp. 1675-1680, Sep. 1993.

[20] L. N. Atallah, J.-P. Barbot, and P. Larzabal, "From Chapman-Robbins bound towards Barankin bound in threshold behaviour prediction," Electronics Letters, vol. 40, no. 4, pp. 279-280, Feb. 2004.

[21] T. L. Marzetta, "Computing the Barankin bound, by solving an unconstrained quadratic optimization problem," in Proc. Int. Conf. Acoustics, Speech, and Signal Processing (ICASSP), vol. 5, Apr. 1997, pp. 38293832.

[22] F. Gustafsson and R. Karlsson, "Generating dithering noise for maximum likelihood estimation from quantized data," Automatica, vol. 49, no. 2, pp. 554-560, Feb. 2013.

[23] D. Jin, F. Yin, C. Fritsche, A. M. Zoubir, and F. Gustafsson, "Dithering in quantized RSS based localization," in Proc. Int. Conf. Computational Advances in Multi-Sensor Adaptive Processing (CAMSAP), 2015.

[24] Y. Zhao, F. Yin, and F. Gunnarsson, "Particle filtering for positioning based on proximity reports," in Proc. Int. Conf. on Information Fusion, Washington, D.C., USA, July 2015, pp. 1046-1052.

[25] N. Bergman, "Recursive Bayesian estimation: Navigation and tracking applications," Ph.D. dissertation, Department of Electrical Engineering, Linköping University (LiU), Linkoping, Sweden, 1999.

[26] S. Raza, P. Misra, T. He, and T. Voigt, "Bluetooth smart: An enabling technology for the Internet of Things," in IEEE Int. Conf. on Wireless and Mobile Computing, Networking and Communications (WiMob), Abu Dhabi, UAE, Oct. 2015, pp. 155-162.

[27] 3GPP, Radio Resource Control (RRC); Protocol specification,3GPP, Radio Resource Control (RRC); Protocol specification,, 3rd Generation Partnership Project (3GPP), TS 25.331. Std. [Online]. Available: http://www.3gpp.org/DynaReport/25331.htm

[28] 3GPP, Evolved Universal Terrestrial Radio Access (E-UTRA); Radio Resource Control (RRC); Protocol specification, 3rd Generation Partnership Project (3GPP), TS 36.331. Std. [Online]. Available: http://www.3gpp.org/DynaReport/36331.htm

[29] F. Yin, "Robust wireless localization in harsh mixed line-of-sight/nonline-of-sight environments," Ph.D. dissertation, Technische Universitaet Darmstadt, Darmstadt, Germany, Aug. 2014

[30] E. Snelson and Z. Ghahramani, "Sparse Gaussian processes using pseudo-inputs," in Proc. Int. Conf. Advances in Neural Information Processing Systems. MIT press, 2006, pp. 1257-1264.

[31] S. Särkkä, Bayesian Filtering and Smoothing. Cambridge University Press., 2013. 